\title{
Quasi cubic trigonometric curve and surface
}

\author{
Zhang Guicang, Wang Kai \\ (College of Mathematics and Statistics, Northwest Normal University, Lanzhou, 730070, China) \\ Correspondence: 616688448@qq.com
}

\begin{abstract}
Firstly, a new set of Quasi-Cubic Trigonometric Bernstein basis with two tension shape parameters is constructed, and we prove that it is an optimal normalized totally basis in the framework of Quasi Extended Chebyshev space. And the Quasi-Cubic Trigonometric Bézier curve is generated by the basis function and the cutting algorithm of the curve are given, the shape features (cusp, inflection point, loop and convexity) of the Quasi-Cubic Trigonometric Bézier curve are analyzed by using envelope theory and topological mapping; Next we construct the non-uniform Quasi-Cubic Trigonometric B-spline basis by assuming the linear combination of the optimal normalized totally positive basis have partition of unity and $C^{2}$ continuity, and its expression is obtained. And the non-uniform B-spline basis is proved to have totally positive and high-order continuity. Finally, the non-uniform Quasi Cubic Trigonometric B-spline curve and surface are defined, the shape features of the non-uniform Quasi-Cubic Trigonometric B-spline curve are discussed, and the curve and surface are proved to be $C^{(2 n-1)}$ continuous.
\end{abstract}

Key words: Quasi Extended Chebyshev space; optimal normalized totally positive basis; high-order continuity; shape preserving; shape features

\section{Introduction}

The construction of basis functions has always been an important topic in computer-aided geometric design (CAGD)and computer graphics(CG). A class of practical basis functions often have an important impact on the development of the geometric industry. Traditional Bernstein and B-spline basis, especially cubic basis functions, are widely used in geometric industrial designs, but the resulting curve positions are relatively fixed to the control polygon. Although the NURBS method has a weighting factor that can adjust the shape of the curve, the effect of the weighting factor on the curve is often unclear to the researchers[1-3].

In order to solve such problems, a large number of new basis functions are constructed. The simplest method is to add shape parameters to the classical Bernstein and B-spline basis[4-8]. But in order to be able to effectively modify the shape of the curve, a lot of work is around the tension parameters [9-14]. Based on the $C^{4}$ splines, the $C^{2} \cap F C^{3}$ spline curve with tension parameters is constructed[15]. On this basis, a group of $C^{2} \cap G^{3}$ spline curve with three shape parameters is constructed[16]. In [17], Han constructed a class of spline curves with two exponential shape parameters which possesses $C^{2} \cap F C^{2 k+3}$ continuity. Given the shape preserving property of the curve, Costantini [18] constructed a set of variable degree polynomial basis in the space $\left\{1, t,(1-t)^{p}, t^{q}\right\}$, which is a Quasi Extended Chebyshev(QEC) space[19,20]. From that, the space $\left\{1, t, t^{2}, \cdots, t^{n-2},(1-t)^{p}, t^{q}\right\}$ is also proved to be a QEC space[21]. Lately, in the framework of Canonical Complete Chebyshev space, it is proved that the variable degree splines basis possesses totally positivity property[22]. In [23], Zhu constructed a kind of quasi Bernstein basis with two shape parameters, which is proved an optimal normalized totally positive basis, and the related B-spline curve possessing $C^{2} \cap F C^{k+3}$ continuous. In [24], Wang constructed a group of DTB-like basis with two denominator shape parameters, and the associated B-spline basis possesses $C^{2 n-1}$ continuity when the shape parameters are globally parameter. In [36], the changeable spline basis is given.

In this paper, the theoretical knowledge of QEC- space is applied to prove that $T_{\alpha, \beta}=\operatorname{span}\left\{1, \sin ^{2} t,(1-\sin t)^{3}(1-\alpha \sin t),(1-\cos t)^{3}(1-\beta \cos t)\right\}$ space with shape parameters constitutes QEC space, and the expression of the optimal normalized totally positive basis(B basis), the definition and corner cutting 
algorithm of Quasi-Cubic Trigonometric Bézier curve(QCT-Bézier), and the shape analysis diagram of QCT-Bézier curve are given. The presented noon-uniform Quasi cubic trigonometric B-spline (QCT-B spline) curves have $C^{2}$ continuity at every knots, which can be $C^{(2 n-1)}(n=0,1,2,3, \cdots)$ continuous for a uniform vector and a shape parameter. The shape parameters of QCT-Bézier curves and QCT-B spline curve and surface can be adjusted foreseeably.

The other work arrangements of this paper are as follows: A class of B basis is given in Section 3. Section 4 presents the definition, corner cutting algorithm and shape features of QCT-Bézier curve. In the section 5, a group of QCT-B spline basis is proposed and its properties are analyzed. Section 6 gives the definition and local adjustable properties of QCT-B spline curve. Section 7 gives the definition and high order continuity of QCT-B spline surfaces. Finally, Section 8 gives the conclusion.

\section{Preliminaries}

In order to better understand this paper, this paper gives some theoretical knowledge about QEC space and Extended Completed Chebyshev(ECC). For more detailed information, please refer to [19, 25-31].

Use $I$ to represent an arbitrarily given $[a, b]$, which has the following definition:

Definition 1 ECC space: If there are 4 positive weight functions $w_{j} \in C^{n-j}(I)(j=0,1,2,3)$, it has the following form

$$
\begin{aligned}
& u_{0}(t)=w_{0}(t), \\
& u_{1}(t)=w_{0}(t) \int_{a}^{t} w_{1}\left(t_{1}\right) d t_{1}, \\
& u_{2}(t)=w_{0}(t) \int_{a}^{t} w_{1}\left(t_{1}\right) \int_{a}^{t_{1}} w_{2}\left(t_{2}\right) d t_{2} d t_{1}, \\
& u_{3}(t)=w_{0}(t) \int_{a}^{t} w_{1}\left(t_{1}\right) \int_{a}^{t_{1}} w_{2}\left(t_{2}\right) \int_{a}^{t_{2}} w_{3}\left(t_{3}\right) d t_{3} d t_{2} d t_{1},
\end{aligned}
$$

we call $\left(u_{0}, u_{1}, u_{2}, u_{3}\right)$ a 4-dimension ECC-space.

4-dimension function space $\left(u_{0}, u_{1}, u_{2}, u_{3}\right)$ is an ECC space on $I$ if and only if for arbitrary $k(0 \leq k \leq 3)$, arbitrary linear combination of the subspace $\left(u_{0}, \cdots, u_{k}\right)$ has at most $k$ zeros (counting multiplicities).

Definition 2 QEC space: 4-dimension function space $\left(u_{0}, u_{1}, u_{2}, u_{3}\right)$ is an QEC space on $I$ if and only if for arbitrary linear combination of the subspace $\left(u_{0}, u_{1}, u_{2}, u_{3}\right)$ has at most 3 zeros (counting multiplicities).

Definition 3 Totally positive basis: Basis functions $\left(u_{0}, u_{1}, u_{2}, u_{3}\right)$ is said to be totally positive on closed bounded interval $[a, b]$ if, for arbitrary sequence of points $a \leq t_{0}<t_{1}<t_{2}<t_{3}<b$, the collocation matrix $\left(u_{j}\left(t_{i}\right)\right)_{0 \leq i, j \leq 3}$ is totally positive ; that is, all its minors are nonnegative.

For a function space with a totally positive basis, the B basis is unique. Other totally positive basis can be deduced by multiplying a totally positive matrix in the basis of B basis.

\section{Construction of $B$ basis}

For any $t \in[0, \pi / 2], \alpha, \beta \in[-1,1]$, we will construct Quasi-Cubic Trigonometric Bernstein basis (QCT Bernstein basis) in space $T_{\alpha, \beta}=\operatorname{span}\left\{1, \sin ^{2} t,(1-\alpha \sin t)(1-\sin t)^{3},(1-\beta \cos t)(1-\cos t)^{3}\right\}$, therefore, the related mother-function is given as follows:

$$
\Phi(t)=\left\{\sin ^{2} t,(1-\alpha \sin t)(1-\sin t)^{3},(1-\beta \cos t)(1-\cos t)^{3}\right\}, t \in[0, \pi / 2] .
$$

It can be known from the theorem 3.1 of [24] that it is only necessary to prove that the related differential space $D T_{\alpha, \beta}=\operatorname{span}\left\{2 \sin t \cos t,-\cos t(3+\alpha-4 \alpha \sin t)(1-\sin t)^{2}, \sin t(3+\beta-4 \beta \cos t)(1-\cos t)^{2}\right\} \quad$ is a 3-dimensional QEC space on a closed bounded interval $t \in[0, \pi / 2]$.

Theorem 1 For arbitrary $\alpha, \beta \in[-1,1], t \in[0, \pi / 2], D T_{\alpha, \beta}$ is a 3-dimensional QEC space.

Proof For arbitrary $\xi_{i} \in R(i=0,1,2)$, we have

$\xi_{0}[2 \sin t \cos t]+\xi_{1}\left\{-\cos t(3+\alpha-4 \alpha \sin t)(1-\sin t)^{2}\right\}+\xi_{2}\left\{\sin t(3+\beta-4 \beta \cos t)(1-\cos t)^{2}\right\}=0$.

For $t=0$, from (2), we can get $\xi_{1}=0$; For $t=\pi / 2$, we can get $\xi_{2}=0$; Finally, we can get $\xi_{0}=0$. 
Therefore, $D T_{\alpha, \beta}$ is a 3-dimension space.

Next, we will prove that $D T_{\alpha, \beta}$ is a 3-dimensional ECC space on $[0, \pi / 2]$. For $t \in[a, b] \subset(0, \pi / 2)$, let

$$
\begin{aligned}
& u(t)=\frac{3+\alpha+\sin ^{2} t(8 \alpha \sin t-3-9 \alpha)}{\sin ^{2} t} \cos t>0, \\
& v(t)=\frac{3+\beta+\cos ^{2} t(8 \beta \cos t-3-9 \beta)}{\cos ^{2} t} \sin t>0, \\
& u^{\prime}(t)=\frac{-6+\alpha\left(-2+8 \sin ^{3} t\right)}{\sin ^{2} t} \cos ^{2} t-\frac{3+\alpha+\sin ^{2} t(8 \alpha \sin t-3-9 \alpha)}{\sin t}<0, \\
& v^{\prime}(t)=-\frac{-6+\beta\left(-2+8 \cos ^{3} t\right)}{\cos ^{3} t} \sin ^{2} t+\frac{3+\beta+\cos ^{2} t(8 \beta \cos t-3-9 \beta)}{\cos t}>0,
\end{aligned}
$$

Therefore, according to Wronskian, we have

$$
W(u, v)(t)=u(t) v^{\prime}(t)-u^{\prime}(t) v(t)>0, \forall t \in[0, \pi / 2]
$$

For $t \in[a, b]$, we define 3 weight functions as follows

$$
\begin{aligned}
& w_{0}(t)=2 \sin t \cos t, \\
& w_{1}(t)=A u(t)+B v(t), \\
& w_{2}(t)=C \frac{W(u, v)(t)}{[A u(t)+B v(t)]^{2}} .
\end{aligned}
$$

Where $A, B, C$ are 3 any positive real numbers. And $w_{i}(t)(i=0,1,2)$ are positive, bounded, and $C^{\infty}$ on $[a, b]$. We consider the following ECC space defined by $w_{i}(t)(i=0,1,2)$,

$$
\begin{aligned}
& u_{0}(t)=w_{0}(t), \\
& u_{1}(t)=w_{0}(t) \int_{a}^{t} w_{1}\left(t_{1}\right) d t_{1}, \\
& u_{2}(t)=w_{0}(t) \int_{a}^{t} w_{1}\left(t_{1}\right) \int_{a}^{t_{1}} w_{2}\left(t_{2}\right) d t_{2} d t_{1} .
\end{aligned}
$$

We can verify that $u_{0}(t), u_{1}(t), u_{2}(t)$ are the linear combination of $2 \sin t \cos t,-\cos t(3+\alpha-4 \alpha \sin t)(1-\sin t)^{2}$, $\sin t(3+\beta-4 \beta \cos t)(1-\cos t)^{2}$. According to[24-29,32], we can obtain that $D T_{\alpha, \beta}$ is a 3-dimensional ECC space on $[a, b]$. In addition, $[a, b]$ are any subinterval of $(0, \pi / 2)$, therefore, $D T_{\alpha, \beta}$ is a 3 -dimensional ECC space on $(0, \pi / 2)$.

Next, we further prove $D T_{\alpha, \beta}$ is a 3-dimensional ECC space on $[0, \pi / 2]$ as well. According to [24-29,32], we only prove that arbitrary nonzero element of $D T_{\alpha, \beta}$ has at most 2 zeroes on $[0, \pi / 2]$. Consider the following nonzero function

$$
F(t)=C_{0}[2 \sin t \cos t]+C_{1}\left[-\cos t(3+\alpha-4 \alpha \sin t)(1-\sin t)^{2}\right]+C_{2}\left[\sin t(3+\beta-4 \beta \cos t)(1-\cos t)^{2}\right]
$$

Where $t \in[0, \pi / 2]$. From the precious proof, we have obtained that $D T_{\alpha, \beta}$ is a 3-dimensional ECC space on $(0, \pi / 2)$. Therefore, $F(t)$ has 2 zeros at most on $(0, \pi / 2)$.

We first assume that $F(t)$ vanishes at 0 , we can get $C_{1}=0$. On this occasion, if $C_{2}=0$, we can find that $F(t)$ has two singular zero at 0 and $\pi / 2$, respectively. If $C_{0}=0$, we can find that $F(t)$ only has a singular zero at 0 ; If $C_{0} C_{2}>0$, we have

$$
\begin{aligned}
F(t) & =C_{0}[2 \sin t \cos t]+C_{2}\left[\sin t(3+\beta-4 \beta \cos t)(1-\cos t)^{2}\right] \\
& =\sin t\left[2 C_{0} \cos t+C_{2}(3+\beta-4 \beta \cos t)(1-\cos t)^{2}\right]
\end{aligned}
$$

On this occasion, we can easily get that $F(t)$ vanishes at 0 , but it does not vanish at $\pi / 2$. For $t \in(0, \pi / 2)$, we have

$$
(3+\beta-4 \beta \cos t)(1-\cos t)^{2}>0 .
$$


Therefore, we can get that $F(t)$ is constant positive or negative on $(0, \pi / 2)$. And we can get that $F(t)$ only have a zeroes. If $C_{0} C_{2}<0$, let

$$
g(t)=2 C_{0} \cos t+C_{2}(3+\beta-4 \beta \cos t)(1-\cos t)^{2} .
$$

Thus, we have

$$
g^{\prime}(t)=\sin t\left\{-2 C_{0}+12 \sin ^{2}(t / 2)((1+\beta) \sin t-\beta \sin 2 t)\right\},
$$

we let $h(t)=\sin ^{2}(t / 2)((1+\beta) \sin t-\beta \sin 2 t)$, we can easily prove that $h(t) \geq 0$. Therefore, we can get that $g(t)$ is monotonically decreasing or monotonically increasing function on $(0, \pi / 2) \cdot g(t)$ has at most 1 zeroes on $(0, \pi / 2)$. Thus, $g(t)$ has at most 2 zeroes on $[0, \pi / 2]$.

From the previous discussion we know that $D T_{\alpha, \beta}$ is a 3-dimension QEC space. By theorem [24] of 3.1, we get that blossom exits $T_{\alpha, \beta}$. Thus, by theorem [24] of 2.13, we can easily deduce that $T_{\alpha, \beta}$ possesses a B basis on $[0, \pi / 2]$.

Theorem 2: For $\alpha, \beta \in[-1,1], t \in[0, \pi / 2]$, the related $\mathrm{B}$ basis of $T_{\alpha, \beta}$ is

$$
\left\{\begin{array}{l}
T_{0}(t)=(1-\sin t)^{3}(1-\alpha \sin t), \\
T_{1}(t)=1-\sin ^{2} t-(1-\sin t)^{3}(1-\alpha \sin t), \\
T_{2}(t)=1-\cos ^{2} t-(1-\cos t)^{3}(1-\beta \cos t), \\
T_{3}(t)=(1-\cos t)^{3}(1-\beta \cos t)
\end{array}\right.
$$

We also call B basis as QCT-Bernstein basis with two parameters $\alpha$ and $\beta$.

Proof: For any $\alpha, \beta \in[-1,1]$, from the (1), we have

$$
\begin{array}{ll}
\Phi(0)=(0,1,0), & \Phi(\pi / 2)=(1,0,1), \\
\Phi^{\prime}(0)=(0,-(\alpha+3), 0), & \Phi^{\prime}(\pi / 2)=(0,0, \beta+3), \\
\Phi^{\prime \prime}(0)=(2,6 \alpha+6,0), & \Phi^{\prime \prime}(\pi / 2)=(-2,0,6 \beta+6) .
\end{array}
$$

From the properties of blossom[16-21], we have

$$
\begin{aligned}
& \Pi_{0}=\Phi(0)=(0,1,0), \Pi_{3}=\Phi(\pi / 2)=(1,0,1), \\
& \left\{\Pi_{1}\right\}=O s c_{1} \Phi(0) \cap O s c_{2} \Phi(\pi / 2)=(0,0,0), \\
& \left\{\Pi_{2}\right\}=O s c_{2} \Phi(0) \cap O s c_{1} \Phi(\pi / 2)=(1,0,0) .
\end{aligned}
$$

From $t \in[0, \pi / 2]$, from $\Phi(t)=\sum_{i=0}^{3} A_{i}(t) \Pi_{i}$, we have

$$
\left\{\begin{array}{l}
T_{2}(t)+T_{3}(t)=\sin ^{2} t, \\
T_{0}(t)=(1-\sin t)^{3}(1-\alpha \sin t), \\
T_{3}(t)=(1-\cos t)^{3}(1-\beta \cos t) .
\end{array}\right.
$$

From $\Phi(t)$ together with $\sum_{i=0}^{3} T_{i}(t)=1$, we can easily deduce the expressions of (3). Next, we verify that $T_{i}(t), i=0,1, \cdots, 3$ is a B basis of $T_{\alpha, \beta}$.

Firstly, we prove that $T_{i}(t), i=0,1, \cdots, 3$ are linear combination. For any $\xi_{i} \in R(i=0,1,2,3)$, we consider the following linear combination:

$$
\sum_{i=0}^{3} \xi_{i} T_{i}(t)=0
$$

And then we differentiate both side of (4), we have

$$
\sum_{i=0}^{3} \xi_{i} T_{i}^{\prime}(t)=0
$$

When $t=0$, feom (4) and (5), we have

$$
\left\{\begin{array}{l}
\xi_{0}=0, \\
(\alpha+3)\left(\xi_{0}-\xi_{1}\right)=0 .
\end{array}\right.
$$


Thus, we can obtain that $\xi_{0}=\xi_{1}=0$. When $t=\pi / 2$, we have $\xi_{2}=\xi_{3}=0$. Thus, we can easily prove $T_{i}(t), i=0,1,2,3$ are linearly independent. We also easily verify $T_{i}(t) \geq 0, i=0,1,2,3$ on $[0, \pi / 2]$ and $T_{i}(t)>0$ on $(0, \pi / 2)$. In addition, we can easily prove that $T_{i}(t) \geq 0, i=0,1,2,3$ possesses the following end-point property:

$T_{0}(0)=1$, and $T_{0}(t)$ vanishes 3 times at $\pi / 2$;

$T_{3}(\pi / 2)=1$, and $T_{3}(t)$ vanishes 3 times at 0 .

Thus, from [24], we can know that QCT-Bernstein basis $T_{i}(t) \geq 0, i=0,1,2,3$ is a B basis of $T_{\alpha, \beta}$.

Fig. 1 gives some plots of QCT-Bernstein basis.
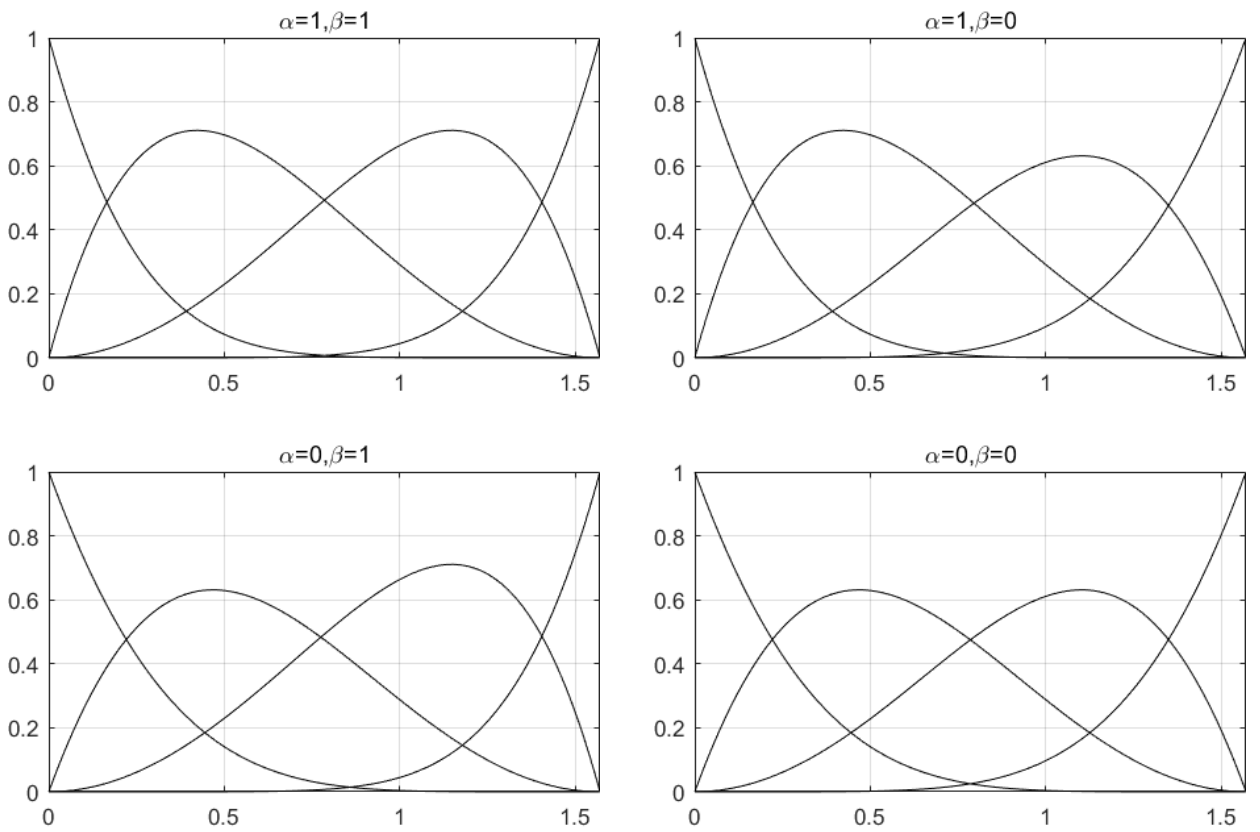

Fig.1 The image of QCT-Bernstein basis with different shape parameters

\section{QCT-Bézier curves}

\subsection{The definition and properties of curve}

Definition 4 Given control points $P_{i}(i=0,1, \ldots, n) \in R^{2} / R^{3}, n \geq 3$. Then

$$
Q(t ; \alpha, \beta)=\sum_{i=0}^{3} T_{i}(t ; \alpha, \beta) P_{i}, t \in[0, \pi / 2], \alpha, \beta \in[-1,1]
$$

is called a QCT-Bézier curves with 2 shape parameters $\alpha$ and $\beta$.

Since QCT-Bernstein basis has the properties of totally positive, nonnegatively and partition of unity, the related QCT-Bézier curves devoted in (6) possesses variation diminishing, affine invariance and convex hull, which means that QCT-Bézier curves are suitable for geometric design. In addition, for any $\alpha, \beta \in[-1,1]$, we have the following end-point property:

$$
\left\{\begin{array}{l}
Q(0 ; \alpha, \beta)=P_{0}, \\
Q(\pi / 2 ; \alpha, \beta)=P_{3}, \\
Q^{\prime}(0 ; \alpha, \beta)=(\alpha+3)\left(P_{1}-P_{0}\right), \\
Q^{\prime}(\pi / 2 ; \alpha, \beta)=(\beta+3)\left(P_{3}-P_{2}\right), \\
Q^{\prime \prime}(0 ; \alpha, \beta)=(6 \alpha+6)\left(P_{0}-P_{1}\right)+2\left(P_{2}-P_{1}\right), \\
Q^{\prime \prime}(\pi / 2 ; \alpha, \beta)=(6 \beta+6)\left(P_{3}-P_{2}\right)+2\left(P_{1}-P_{2}\right) .
\end{array}\right.
$$

From the properties discussed above, we know that the QCT-Bézier curves keep all the properties of traditional Bézier curves. Since QCT-Bézier curves have 2 parameters $\alpha$ and $\beta$, it has the property of flexible shape adjustability.

\subsection{Shape adjustment of QCT-Bézier curves}


Next, we rewrite (6) as the following form:

$$
T(t ; \alpha, \beta)=P_{1} \cos ^{2} t+P_{2} \sin ^{2} t+T_{0}(t ; \alpha)\left(P_{0}-P_{1}\right)+T_{3}(t ; \beta)\left(P_{3}-P_{2}\right) .
$$

Obviously, for any settled $t \in(0, \pi / 2), T_{0}(t ; \alpha)$ decreases with the increase of $\alpha$, which indicates that the QCT-Bézier curve has the same direction of the edge vector $P_{0}-P_{1}$ as $\alpha$ increases. When $\alpha$ decreases, the situation is just the opposite. For edge vector $P_{3}-P_{2}, \beta$ has the same effect. When $\alpha$ or $\beta$ increases, QCT-Bézier curves will approach $P_{2}$ and $P_{3}$, respectively. When $\alpha=\beta$ increases or decreases, QCT-Bézier curve has the opposite or same direction of the edge vector $P_{2}-P_{1}$, which indicates that parameters $\alpha$ and $\beta$ have the tension effect. Fig.2 shows the QCT-Bézier curves with different shape parameters.

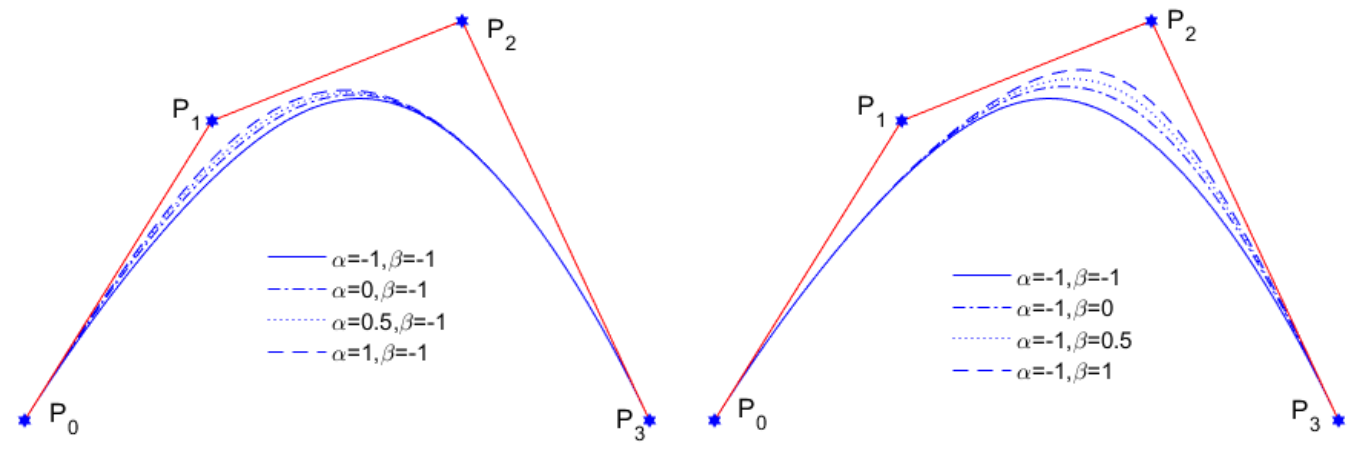

Fig.2 The adjustment of the QCT-Bézier curves

\subsection{The corner cutting algorithm}

Below, we develop a corner cutting algorithm to effectively calculate QCT-Bézier curves. To this end, the QCT-Bézier curves are written in the matrix form of (8). Fig.3 shows the whole process of calculating QCT-Bézier curves.

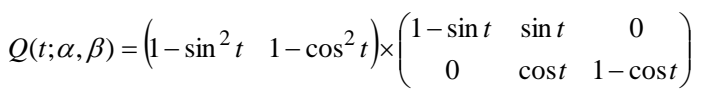

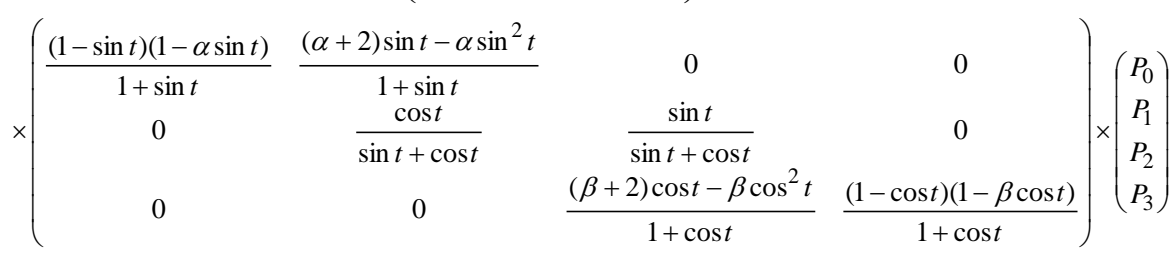
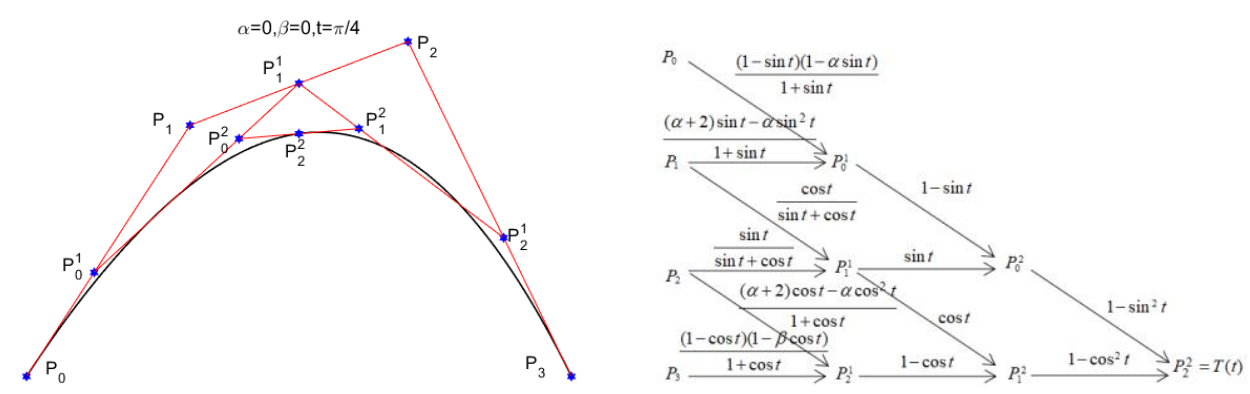

Fig.3 The corner cutting algorithm

\subsection{The shape analysis of QCT-Bézier curves}

In this section, we will use envelope theory and topological mapping to describe the shape features of the proposed QCT-Bézier curve given in (6). The relevant cusps, inflection points, loops, convexity as well as envelope and topological mapping theories can be referred to [33-35].

4.4.1 The shape analysis of spatial QCT-Bézier curve

Theorem 3 When $\alpha, \beta \in[-1,1], t \in[0, \pi / 2]$, if $P_{i}(i=0,1,2,3)$ are not coplanar, QCT-Bézier curve is spatial 
curve, it does not have cusps, loops and inflection points, and it has the same rotation direction as $P_{i}(i=0,1,2,3)$.

Proof: Let $a_{i}=P_{i}-P_{i-1}, i=1,2,3$, we rewrite $Q(t)(6)$ as

$$
Q(t)=P_{0}+\left[1-T_{0}(t ; \alpha)\right] a_{1}+\left[T_{2}(t ; \beta)+T_{3}(t ; \beta)\right] a_{2}+T_{3}(t ; \beta) a_{3} .
$$

Thus, we have

$$
Q^{\prime}(t)=-T_{0}^{\prime}(t ; \alpha) a_{1}+\left[T_{2}^{\prime}(t ; \beta)+T_{3}^{\prime}(t ; \beta)\right] a_{2}+T_{3}^{\prime}(t ; \beta) a_{3} .
$$

When $0<t<\pi / 2$, according (3), we have $T_{2}^{\prime}(t ; \beta)+T_{3}^{\prime}(t ; \beta)=2 \sin t \cos t \neq 0$. According to $P_{i}(i=0,1,2,3)$ are not coplanar, we can know that the vector $a_{i}(i=1,2,3)$ are not linear combination, thus, $Q(t) \neq 0$, and it has not cusps.

Next, we assume that $Q(t)$ possesses loops, when $0 \leq t_{1}<t_{2} \leq \pi / 2$, we have $Q\left(t_{1}\right)-Q\left(t_{2}\right)=0$. Thus,

$\left[T_{0}\left(t_{2} ; \alpha\right)-T_{0}\left(t_{1} ; \alpha\right)\right] a_{1}+\left[T_{2}\left(t_{1} ; \beta\right)+T_{3}\left(t_{1} ; \beta\right)-T_{2}\left(t_{2} ; \beta\right)-T_{3}\left(t_{2} ; \beta\right)\right] a_{2}+\left[T_{3}\left(t_{1} ; \beta\right)-T_{3}\left(t_{2} ; \beta\right)\right] a_{3}=0$. According precious discussion, we have known that $a_{i}(i=1,2,3)$ are linear independent, thus, from (10), we have $T_{0}\left(t_{1} ; \alpha\right)=T_{0}\left(t_{2} ; \alpha\right) ; T_{2}\left(t_{1} ; \beta\right)=T_{2}\left(t_{2} ; \beta\right) ; T_{3}\left(t_{1} ; \beta\right)=T_{3}\left(t_{2} ; \beta\right)$. Then, we let $T_{0}^{\prime}(t ; \alpha)=0$, we have $-\cos t(3+\alpha-4 \alpha \sin t)(1-\sin t)^{2}=0$, we can obtain $t=\pi / 2(\times)$ and $t=\arcsin ((\alpha+3) / 4 \alpha)$. According to $0<\arcsin ((\alpha+3) / 4 \alpha)<\pi / 2$, we have $\alpha<-3$ or $\alpha>1$. Thus, when $\alpha \in[-3,1], T_{0}(t ; \alpha)$ is monotonically decreasing on $[0, \pi / 2], T_{0}(t ; \alpha)$ has not loops. Thus, when $\alpha \in[-1,1], Q(t)$ has not loops.

Then, let $G(t)=\operatorname{det}\left(Q^{\prime}(t), Q^{\prime \prime}(t), Q^{\prime \prime \prime}(t)\right)$, we have

$$
\begin{aligned}
& G(t)=\operatorname{det}\left[\sum_{i=0}^{3} P_{i} T_{i}^{\prime}(t) \quad \sum_{i=0}^{3} P_{i} T_{i}^{\prime \prime}(t) \quad \sum_{i=0}^{3} P_{i} T_{i}^{\prime \prime}(t)\right] \\
& =\left|\begin{array}{cccc}
\sum_{i=0}^{3} T_{i}(t) & \sum_{i=0}^{3} T_{i}^{\prime}(t) & \sum_{i=0}^{3} T_{i}^{\prime \prime}(t) & \sum_{i=0}^{3} T_{i}^{\prime \prime}(t) \\
\sum_{i=0}^{3} P_{i} T_{i}(t) & \sum_{i=0}^{3} P_{i} T_{i}^{\prime}(t) & \sum_{i=0}^{3} P_{i} T_{i}^{\prime \prime}(t) & \sum_{i=0}^{3} P_{i} T_{i}^{\prime \prime}(t)
\end{array}\right| \\
& =\left\lfloor\left[\begin{array}{cccc}
1 & 1 & 1 & 1 \\
P_{0} & P_{1} & P_{2} & P_{3}
\end{array}\right]\left[\begin{array}{cccc}
T_{0}(t) & T_{0}^{\prime}(t) & T_{0}^{\prime \prime}(t) & T_{0}^{\prime \prime \prime}(t) \\
T_{1}(t) & T_{1}^{\prime}(t) & T_{1}^{\prime \prime}(t) & T_{1}^{\prime \prime}(t) \\
T_{2}(t) & T_{2}^{\prime}(t) & T_{2}^{\prime \prime}(t) & T_{2}^{\prime \prime \prime}(t) \\
T_{3}(t) & T_{3}^{\prime}(t) & T_{3}^{\prime \prime}(t) & T_{3}^{\prime \prime \prime}(t)
\end{array}\right] \mid\right. \\
& =\left\lfloor\left[\begin{array}{cccc}
1 & 0 & 0 & 0 \\
P_{0} & a_{1} & a_{2} & a_{3}
\end{array}\right]\left[\begin{array}{cccc}
T_{0}(t) & T_{0}^{\prime}(t) & T_{0}^{\prime \prime}(t) & T_{0}^{\prime \prime \prime}(t) \\
T_{1}(t) & T_{1}^{\prime}(t) & T_{1}^{\prime \prime}(t) & T_{1}^{\prime \prime}(t) \\
T_{2}(t) & T_{2}^{\prime}(t) & T_{2}^{\prime \prime}(t) & T_{2}^{\prime \prime \prime}(t) \\
T_{3}(t) & T_{3}^{\prime}(t) & T_{3}^{\prime \prime}(t) & T_{3}^{\prime \prime \prime}(t)
\end{array}\right] \mid\right. \\
& =\left(a_{1}, a_{2}, a_{3}\right) D(t)
\end{aligned}
$$

Where, $\left(a_{1}, a_{2}, a_{3}\right)$ is mixed product of vector edge $a_{1}, a_{2}, a_{3}$, by directly computing, we have $D(t)>0$. For any $0<t<\pi / 2$, from $\left(a_{1}, a_{2}, a_{3}\right) \neq 0$, we have $G(t) \neq 0$, and it has same positive and negative property as $\left(a_{1}, a_{2}, a_{3}\right)$. Thus, $Q(t)$ has not inflection points, and it has the same rotation direction as the control points.

4.4.2 The shape analysis of the planar QCT-Bézier curve

If $P_{i}(i=0,1,2,3)$ are coplanar, $Q(t)$ is planar curve, at this time, we have $\left(a_{1}, a_{2}, a_{3}\right)=0$. Firstly, we consider $a_{1} \| a_{3}$, we have $a_{2}=u a_{1}+v a_{3}$. Substituting it into (9), we have

$$
Q(t)=P_{0}+\left[1-T_{0}(t)+u\left(T_{2}(t)+T_{3}(t)\right)\right] a_{1}+\left[T_{3}(t)+v\left(T_{2}(t)+T_{3}(t)\right)\right] a_{3}
$$

1) Cusps

The necessary condition that the planar QCT-Bézier curve $Q(t)$ has cups is $Q^{\prime}(t)=0(0<t<\pi / 2)$. From (11), we have

$$
\left[-T_{0}^{\prime}(t)+u\left(T_{2}^{\prime}(t)+T_{3}^{\prime}(t)\right)\right] a_{1}+\left[T_{3}^{\prime}(t)+v\left(B_{2}^{\prime}(t)+B_{3}^{\prime}(t)\right)\right] a_{3}=0
$$

Because $a_{1}$ and $a_{3}$ are linear independent, from (12) and (3), we have 


$$
C:\left\{\begin{array}{l}
u=\frac{(1-\sin t)^{2}(-3-\alpha+4 \alpha \sin t)}{2 \sin t} \\
v=-\frac{(1-\cos t)^{2}(3+\beta-4 \beta \cos t)}{2 \cos t}
\end{array}, 0<t<\pi / 2\right.
$$

We analyze the shape of $C$, from (13), we have

$$
\begin{aligned}
& \lim _{t \rightarrow 0} u=-\infty, \lim _{t \rightarrow 0} v=0 \\
& \lim _{t \rightarrow \pi / 2} u=0, \lim _{t \rightarrow \pi / 2} v=-\infty
\end{aligned}
$$

This shows that $C$ has two asymptotes $u=0$ and $v=0$, respectively. For any $\alpha, \beta \in[-1,1]$, we have $\frac{d u}{d v}<0$, $\frac{d^{2} u}{d u^{2}}>0$. This indicates that the $C$ is a monotonically decreasing and strictly convex curve. For any $\left(u_{0}, v_{0}\right) \in C$, we have $Q^{\prime}(t)=0$ and $Q^{\prime \prime}(t) \neq 0$. In fact, similar to the discussion of (12) and (13), we have

$$
\begin{aligned}
& {\left[-T_{0}^{\prime \prime}(t)+u\left(T_{2}^{\prime \prime}(t)+T_{3}^{\prime \prime}(t)\right)\right] a_{1}+\left[T_{3}^{\prime \prime}(t)+v\left(T_{2}^{\prime \prime}(t)+T_{3}^{\prime \prime}(t)\right)\right] a_{3}=0} \\
& \left\{\begin{array}{l}
u=\frac{[\cos (t / 2)-\sin (t / 2)]^{4}[6-2 \alpha+8 \alpha \cos 2 t+(9-5 \alpha) \sin t]}{2 \cos 2 t} \\
v=\frac{-4((5 \beta-9) \cos t+2(-3+\beta+4 \beta \cos 2 t)) \sin ^{4}(t / 2)}{2 \cos 2 t}
\end{array} \quad 0<t<\pi / 2\right.
\end{aligned}
$$

For any $\alpha, \beta \in[-1,1]$, we can easily verify that (13) and (15) cannot be established at the same time. It indicates $Q^{\prime}(t)=0$ and $Q^{\prime \prime}(t) \neq 0$. Thus, we have

$$
Q^{\prime}(t)=Q^{\prime \prime}(t)\left(t-t_{0}\right)+o\left(t-t_{0}\right) .
$$

When the parameter $t$ passes $t_{0}$, the direction of $Q^{\prime}(t)$ will changes. We can easily conclude that $Q\left(t_{0}\right)$ is a cusp. Therefore, the planar QCT-Bézier curve defined by (11) possess cusps are equivalent to $(u, v) \in C$.

2) Inflection points

From the previous discussion, we have $Q^{\prime}(t) \times Q^{\prime \prime}(t)=f(t, u, v) a_{1} \times a_{3}$. where

$$
f(t ; u, v)=-\left|\begin{array}{cc}
T_{0}^{\prime}(t) & T_{3}^{\prime}(t) \\
T_{0}^{\prime \prime}(t) & T_{3}^{\prime \prime}(t)
\end{array}\right|+u\left|\begin{array}{cc}
T_{2}^{\prime}(t) & T_{3}^{\prime}(t) \\
T_{2}^{\prime \prime}(t) & T_{3}^{\prime \prime}(t)
\end{array}\right|+v\left|\begin{array}{cc}
T_{0}^{\prime}(t) & T_{1}^{\prime}(t) \\
T_{0}^{\prime \prime}(t) & T_{1}^{\prime \prime}(t)
\end{array}\right| .
$$

$Q\left(t_{0}\right)\left(0<t_{0}<\pi / 2\right)$ is an inflection point if and only if $f(t ; u, v)$ change sign at $t_{0}$. In the $u v$-plane, the possible region of inflection points must be covered by the family of straight lines $f(t ; u, v)=0$. According to [33], the curve $C$ is just the envelope of the family of straight lines. From the previous analysis, it can be known that the curve $C$ is a strictly convex continuous curve. Thus, the area swept by the tangent line of the curve $C$ is $S \cup D \cup C$, that is, the area where the inflection point may occur. In Fig. 4 , ' $S$ ' represents the area where the QCT-Bézier curve has one single inflection point; ' $N_{0}, N_{1}, N_{2}$ ' represents the area where the QCT-Bézier curve without inflection points and loops; and ' $D$ ' represents the area where the QCT-Bézier curve with two inflection points; and ' $L$ ' represents the area where the QCT- Bézier curve has loops. 


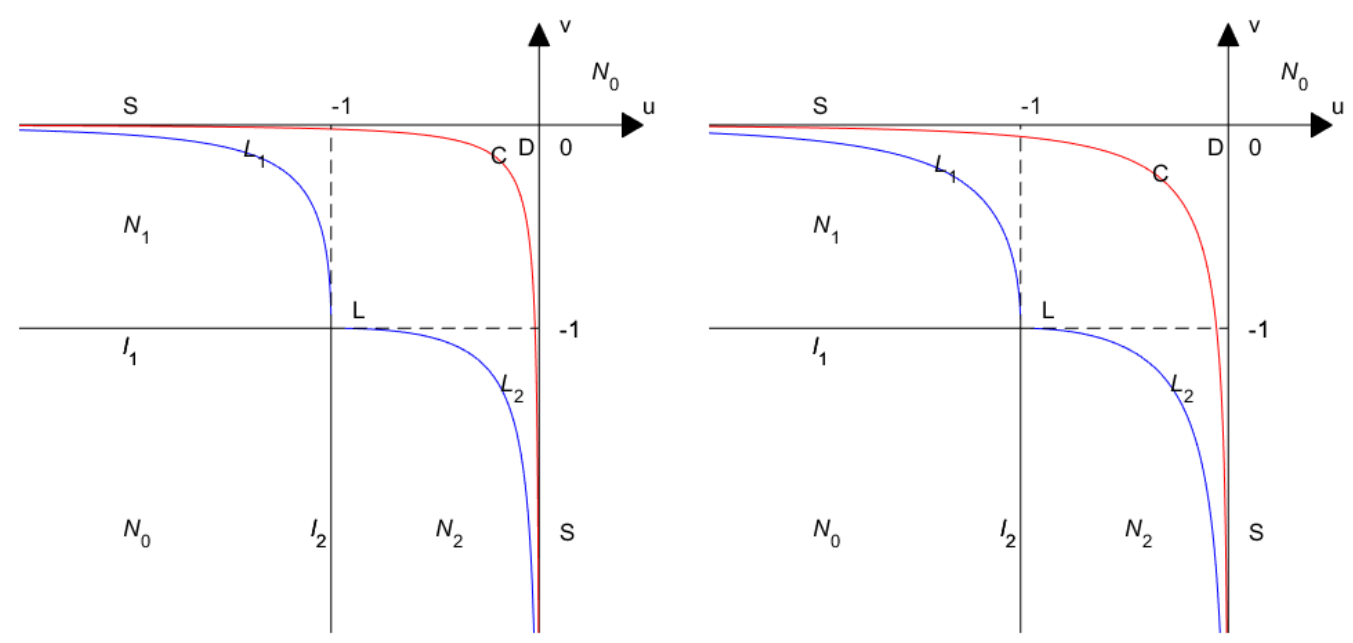

Fig. 4 The shape diagram of QCT-Bézier curve(left: $\alpha=\beta=0$; right: $\alpha=\beta=-1$ )

Obviously, the curve $C$ has at least a tangent line $f\left(t_{0} ; u, v\right)=0$ passing through an any point $\left(u_{0}, v_{0}\right) \in S \cup D \cup C$. When $\left(u_{0}, v_{0}\right) \in C$, according to the Taylor expansion, we have

$$
f\left(t ; u_{0}, v_{0}\right)=\frac{1}{2} f_{t t}^{\prime \prime}\left(t_{0} ; u_{0}, v_{0}\right)\left(t-t_{0}\right)^{2}+o\left(\left(t-t_{0}\right)^{2}\right),
$$

where $f^{\prime \prime}\left(t ; u_{0}, v_{0}\right) \neq 0$. Thus, we can easily conclude that $f\left(t, u_{0}, v_{0}\right)$ does not changes sign at $t_{0}$, the planar QCT-Bézier curve has not inflection points. When $\left(u_{0}, v_{0}\right) \in S \cup D$, we assume that $f\left(t, u_{0}, v_{0}\right)$ is one of tangent line of $C$, which passes $\left(u_{0}, v_{0}\right)$, according to Taylor expansion, we have

$$
f\left(t ; u_{0}, v_{0}\right)=\frac{1}{2} f_{t}^{\prime}\left(t_{0} ; u_{0}, v_{0}\right)\left(t-t_{0}\right)+o\left(t-t_{0}\right) .
$$

We can easily get $f_{t}^{\prime}\left(t_{0}, u_{0}, v_{0}\right) \neq 0$ (if $f_{t}^{\prime}\left(t_{0}, u_{0}, v_{0}\right)=0$, from the definition of envelope, we can know that $\left.\left(u_{0}, v_{0}\right) \in C\right)$. Thus, $f\left(t ; u_{0}, v_{0}\right)$ will change sign at $t_{0}$, the planar QCT-Bézier curve $Q(t)$ has a inflection point at $t_{0}$. In addition, when $\left(u_{0}, v_{0}\right) \in S$, the curve $C$ only has a tangent line which passes $\left(u_{0}, v_{0}\right)$, the related curve $Q(t)$ only has one inflection point; When $\left(u_{0}, v_{0}\right) \in D$, the curve $C$ has two tangent line which passes $\left(u_{0}, v_{0}\right)$, the related curve $Q(t)$ has two inflection points.

3) Loops

The planar QCT-Bézier curve $Q(t)$ has loops if and only if there exists $0 \leq t_{1}<t_{2} \leq \pi / 2$ such that $Q\left(t_{1}\right)=Q\left(t_{2}\right)$. This is equivalent to the parameter $u, v, t_{1}, t_{2}$ satisfying the following equations:

$$
\left\{\begin{array}{l}
u=\frac{T_{0}\left(t_{2} ; \alpha\right)-T_{0}\left(t_{1} ; \alpha\right)}{T_{2}\left(t_{2} ; \beta\right)+T_{3}\left(t_{2} ; \beta\right)-T_{2}\left(t_{1} ; \beta\right)-T_{3}\left(t_{1} ; \beta\right)}, \\
v=\frac{T_{3}\left(t_{1} ; \beta\right)-T_{3}\left(t_{2} ; \beta\right)}{T_{2}\left(t_{2} ; \beta\right)+T_{3}\left(t_{2} ; \beta\right)-T_{2}\left(t_{1} ; \beta\right)-T_{3}\left(t_{1} ; \beta\right)},
\end{array}\left(t_{1}, t_{2}\right) \in \Omega .\right.
$$

Where $\Omega=\left\{\left(t_{1}, t_{2}\right) \in R^{2} \mid 0 \leq t_{1}<t_{2}<\pi / 2\right\}$. Easy to verify (17) defines a topological mapping $F: \Omega \subset R^{2} \rightarrow F(\Omega) \subset R^{2}$, thus, the image $L=F(\Omega)$ is a simply connected region in $u v$-plane. The three boundary lines of $L$ correspond to the three boundary lines $t_{1}=t_{2}, t_{1}=0$ and $t_{2}=1$ of $\Omega$, i.e. the curve $C$ (not belonging to $L$ ), $L_{1}$ and $L_{2}$ (both belonging to $L$ )(see Fig.4). The planar QCT-Bézier curve corresponding to the point in $L$ only has one loop, where the parametric equation of $L_{1}$ and $L_{2}$ are as follows:

$$
L_{1}:\left\{\begin{array}{l}
u=\frac{(1-\alpha \sin t)(1-\sin t)^{3}-1}{\sin ^{2} t}, \\
v=-\frac{(1-\beta \cos t)(1-\cos t)^{3}}{\sin ^{2} t},
\end{array}, \quad 0 \leq t<\pi / 2,\right.
$$




$$
L_{2}:=\left\{\begin{array}{l}
u=-\frac{(1-\alpha \sin t)(1-\sin t)^{3}}{\cos ^{2} t}, \\
v=\frac{(1-\beta \cos t)(1-\cos t)^{3}-1}{\cos ^{2} t}, 0 \leq t<\pi / 2,
\end{array}\right.
$$

From the mathematical analysis, it can be deduced that the curve $L_{1}$ and $L_{2}$ are both monotonically decreasing and strictly convex continuous functions. $L_{1}$ and $L_{2}$ intersect at point $(-1,-1), L_{1}$ with the $u$ axis as the asymptotic line, and $L_{2}$ with the $v$ axis as the asymptotic line. The curve of $C$ does not intersect either $L_{1}$ or $L_{2}$.

4) Convexity

Let $N=R^{2} \backslash(C \cup S \cup D \cup L)$, where the upper left part enclosed by the curve $L_{1}, L_{2}$ (excluding the boundary of $\left.L_{1}, L_{2}\right)$ is $N_{1}$, the lower right part is $N_{2}$, and $N_{0}=N \backslash\left(N_{1} \cup N_{2}\right)$, see Fig.4. From the previous discussion, when $(u, v) \in N$, the planar QCT-Bézier curve $Q(t)$ has not cusps, inflection points and loops. Next, we consider the following vector $M(t)$ and $N(t)$ :

$$
\left\{\begin{array}{l}
M(t)=Q^{\prime}(0) \times[Q(t)-Q(0)], \\
N(t)=[Q(t)-Q(0)] \times Q^{\prime}(t) .
\end{array}\right.
$$

According to (11) and (20), by direct computing, we have

$$
\left\{\begin{array}{l}
M(t)=\xi(t ; u, v)\left(a_{1} \times a_{3}\right), \\
N(t)=\zeta(t ; u, v)\left(a_{1} \times a_{3}\right) .
\end{array}\right.
$$

Where,

$$
\begin{aligned}
& \xi(t ; u, v)=(\alpha+3)\left\{T_{3}(t)+v\left[T_{2}(t)+T_{3}(t)\right]\right\}, \\
& \zeta(t ; u, v)=\left[1-T_{0}(t)\right] T_{3}^{\prime}(t)+T_{3}(t) T_{0}^{\prime}(t)+u\left[T_{2}(t) T_{3}^{\prime}(t)-T_{2}^{\prime}(t) T_{3}(t)\right] \\
& +v\left\{\left[1-T_{0}(t)\right]\left[T_{2}^{\prime}(t)+T_{3}^{\prime}(t)\right]+T_{0}^{\prime}(t)\left[T_{2}(t)+T_{3}(t)\right]\right\} .
\end{aligned}
$$

When $v_{0}=-T_{3}\left(t_{0}\right) /\left[T_{2}\left(t_{0}\right)+T_{3}\left(t_{0}\right)\right], \xi(t ; u, v)$ changes the direction at $t_{0}$. We can easily get $-1<v_{0}<0$. Thus, when $(u, v) \in N_{1}$ (see Fig.4), the curve $Q(t)$ is locally convex[33]. In fact, $N_{1}$ happens to be part of $N$ of the region covered by the tangent line to $L_{2}$. According to literature [33], the envelope of the family of lines $\zeta(t ; u, v)=0$ are exactly curve $L_{1}$, and the region swept by the tangent line of $L_{1}$ in $N$ is $N_{2}$ (see Fig.4). Therefore, when $(u, v) \in N_{2}, Q(t)$ is locally convex. When $(u, v) \in N_{0}=N \backslash\left(N_{1} \cup N_{2}\right), Q(t)$ is globally convex.

Finally, when $a_{1} \| a_{3}$, the curve $Q(t)$ has not cusps and loops; When $a_{1}$ has the same direction of $a_{3}$ (Does not include the 4-point collinear singular case), the curve $Q(t)$ only has a inflection point.

Theorem 4 When $a_{1} \| a_{3}$, the planar QCT-Bézier curve $Q(t)$ has not cusps and loops; If and only if $a_{1}$ has the same direction of $a_{3}, Q(t)$ only has one inflection point; When $a_{1} \| a_{3}$, let $a_{2}=u a_{1}+v a_{3}$, then the shape feature of $Q(t)$ depends on the following distribution of points $(u, v)$ in the $u v$-plane (see Table 1), ie

Table 1 Distribution of shape features of the planar QCT-Bézier curve

\begin{tabular}{ccccc}
\hline \multirow{2}{*}{$(u, v)$} & \multicolumn{4}{c}{ The shape features of the planar QCT-Bézier curve } \\
\cline { 2 - 5 } & Convexity & Cusps & Loops & Inflection points \\
\hline$C$ & & 1 & No & No \\
$S$ & & No & 1 & No \\
$D$ & No & No & No & 1 \\
$N_{0}$ & Global convex & No & No & No \\
$N_{1} \cup N_{2}$ & Locally convex & No & No & No \\
\hline
\end{tabular}

5) Adjusting effect of shape parameters 
Through the above analysis of the shape of the QCT-Bézier curve, the following conclusions can be drawn:

(1) As shown in Fig.5, the change of the shape parameter $\alpha$ and $\beta$ does not affect the single inflection point region $S$ and the global convex region $N_{0}$, so when $Q(t)$ is global convex, it cannot be eliminated by adjusting parameters; and when it is global convex, the shape parameters $(-1<\alpha, \beta \leq 1)$ are modified anyway, the curve is still global convex.

(2) As the shape parameters $\alpha$ and $\beta$ increase, the curve $C$ is stretched toward the point $(0,0), L_{1}$ is stretched toward the point $(-1,0), L_{2}$ is stretched toward the point $(0,-1)$, double inflection points region $D$ shrinks, loops region $L$ expands correspondingly, and locally convex regions $N_{1}$ and $N_{2}$ expand, as shown in Fig.5.

(3) When $(u, v) \in\{(u, v) \mid-1 \leq u, v<0\} \backslash\{(-1,-1)\}$, i.e. when the first and last two edges of the polygon are intersected (except the first and last points coincide), the curve $Q(t)$ may has cusps and loops or inflection points. The curve $Q(t)$ cannot be made into a locally convex curve simply by modifying the shape parameter, as shown in Fig. 6.

(4) When $(u, v) \in\{(u, v) \mid u<-1,-1<v\} \cup\{(u, v) \mid-1<u, v<-1\}$,i.e. when the control polygon is locally convex, the cusps, inflection points and loops of the curve $Q(t)$ can be eliminated by modifying the shape parameter, and the curve can be adjusted to a locally convex curve, as shown in Fig.7.

\section{Non-uniform QCT-B spline basis}

\subsection{The construction of QCT-B spline basis}

Given knots $u_{0}<u_{1}<\cdots<u_{n+4}$, we denote a knot vector with $U=\left(u_{0}, u_{1}, \cdots, u_{n+4}\right)$. Let $h_{j}=u_{j+1}-u_{j}$, $t_{j}(u)=\pi\left(u-u_{j}\right) / 2 h_{j}, j=0,1, \cdots, n+3$, for any real numbers $\alpha_{i}, \beta_{i} \in[-1,1], i=0,1, \cdots, n$. The related QCT-B spline basis is constructed as follows:
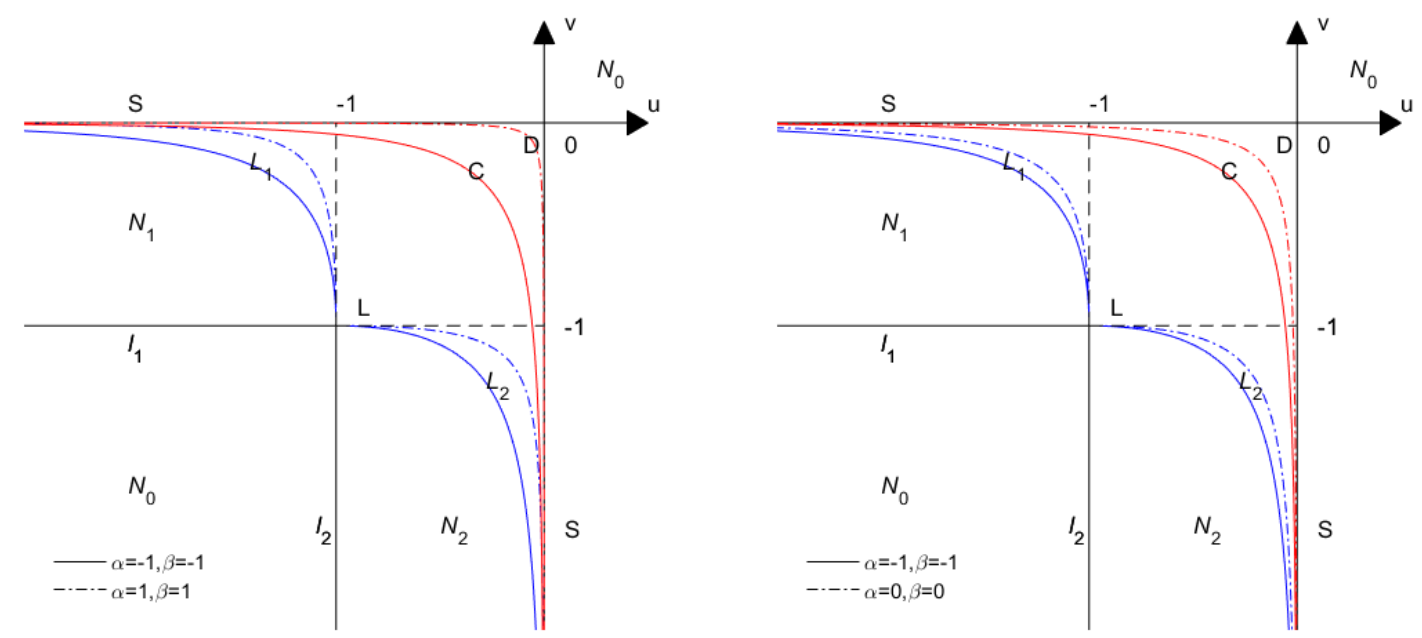


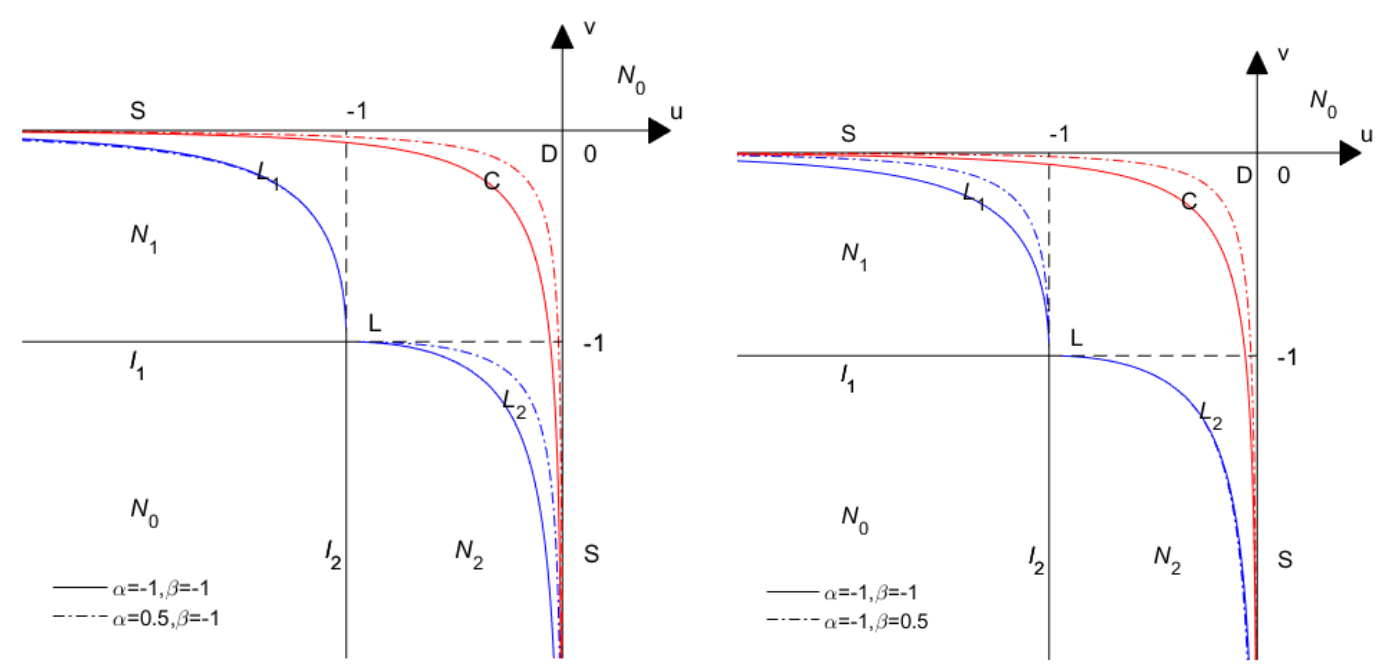

Fig.5 The shape parameter diagram of the QCT-Bézier curve

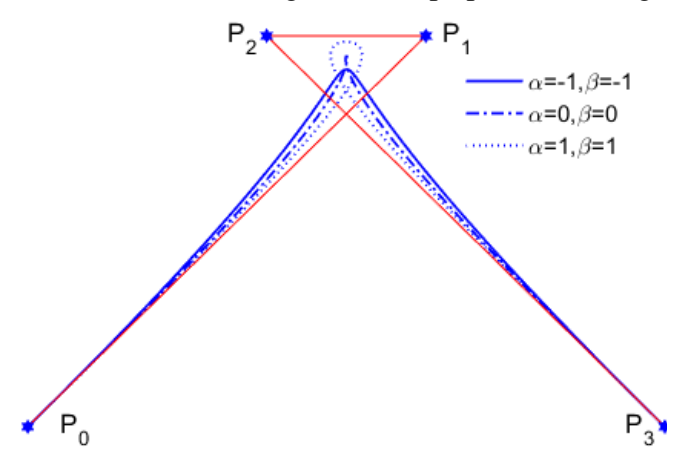

Fig.6 The curve must have a

cusp and loop or a double inflection point

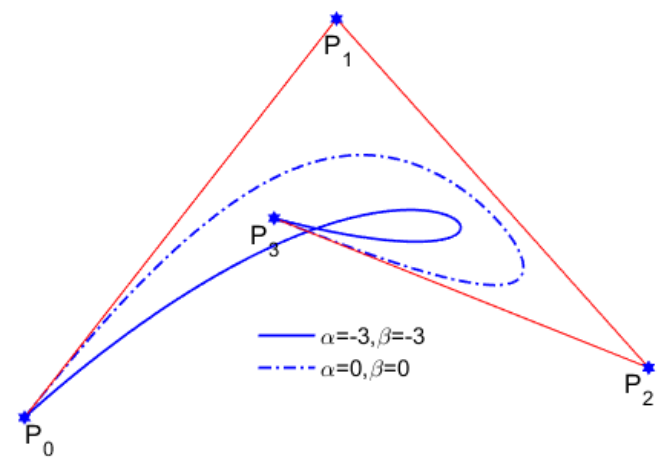

Figure 7 Eliminate double inflection

points, cusp or loop to adjust the curve to local convex

$$
B_{i}(u)= \begin{cases}B_{i, 0}\left(t_{i}\right)=d_{i} T_{3}\left(t_{i} ; \beta_{i}\right), & u \in\left[u_{i}, u_{i+1}\right), \\ B_{i, 1}\left(t_{i+1}\right)=\sum_{j=0}^{3} c_{i+1, j} T_{j}\left(t_{i+1}, \alpha_{i+1}, \beta_{i+1}\right), & u \in\left[u_{i+1}, u_{i+2}\right), \\ B_{i, 2}\left(t_{i+2}\right)=\sum_{j=0}^{3} b_{i+2, j} T_{j}\left(t_{i+2}, \alpha_{i+2}, \beta_{i+2}\right), & u \in\left[u_{i+2}, u_{i+3}\right), \\ B_{i, 3}\left(t_{i+3}\right)=a_{i+3} T_{0}\left(t_{i+3} ; \alpha_{i+3}\right), & u \in\left[u_{i+3}, u_{i+4}\right), \\ 0, & u \notin\left[u_{i}, u_{i+4}\right),\end{cases}
$$

where $T_{j}\left(t_{i} ; \alpha_{i}, \beta_{i}\right), j=0,1,2,3$ is the QCT-Bernstein basis given in equation (3).

In order to determine the coefficient $a_{i}, b_{i, j}, c_{i, j}, d_{i}$, two constraints are given to the constructed QCT-B spline basis: (1) $B_{i}(u)$ has $C^{2}$ continuity on all knots; (2) $B_{i}(u)$ has unity in the interval $\left[u_{3}, u_{n+1}\right]$. After that, you can directly find the coefficient as:

$$
\begin{aligned}
& \lambda_{i}=3\left(\alpha_{i+1}+1\right)\left(\beta_{i}+3\right) h_{i}+3\left(\alpha_{i+1}+3\right)\left(\beta_{i}+1\right) h_{i+1} \\
& \mu_{i}=\left(\alpha_{i+1}+3\right) h_{i}+\left(\beta_{i}+3\right) h_{i+1} \\
& \phi_{i}=\frac{\lambda_{i} h_{i}+\mu_{i} h_{i+1}}{\left(\beta_{i}+3\right) h_{i+1}^{2}} \quad \varphi_{i}=\frac{\lambda_{i-1} h_{i}+\mu_{i-1} h_{i-1}}{\left(\alpha_{i}+3\right) h_{i-1}^{2}} \\
& a_{i}=\frac{\left(\beta_{i-1}+3\right) \lambda_{i-2} h_{i}^{2}}{\lambda_{i-1} \mu_{i-2} h_{i-2}+\lambda_{i-2} \lambda_{i-1} h_{i-1}+\lambda_{i-2} \mu_{i-1} h_{i}} \\
& d_{i}=\frac{\left(\alpha_{i+1}+3\right) \lambda_{i+1} h_{i}^{2}}{\lambda_{i+1} \mu_{i} h_{i}+\lambda_{i} \lambda_{i+1} h_{i+1}+\lambda_{i} \mu_{i+1} h_{i+2}}
\end{aligned}
$$




$$
\begin{aligned}
& b_{i, 0}=\frac{\left(\alpha_{i}+3\right) \phi_{i} h_{i-1}}{\mu_{i-1}} a_{i+1}+\frac{\left(\beta_{i-1}+3\right) \varphi_{i-1} h_{i}}{\mu_{i-1}} d_{i-2} \\
& b_{i, 1}=\phi_{i} a_{i+1} \quad c_{i, 0}=d_{i-1} \\
& b_{i, 2}=\frac{\mu_{i}}{\left(\beta_{i}+3\right) h_{i+1}} a_{i+1} \quad c_{i, 1}=\frac{\mu_{i-1}}{\left(\alpha_{i}+3\right) h_{i-1}} d_{i-1} \\
& b_{i, 3}=a_{i+1} \quad c_{i, 2}=\varphi_{i} d_{i-1} \\
& c_{i, 3}=\frac{\left(\alpha_{i+1}+3\right) \phi_{i+1} h_{i}}{\mu_{i}} a_{i+2}+\frac{\left(\beta_{i}+3\right) \varphi_{i} h_{i+1}}{\mu_{i}} d_{i-1}
\end{aligned}
$$

Definition 5 For any $\alpha_{i}, \beta_{i} \in[-1,1]$, given kont vector $U$ and coefficient $a_{i}, b_{i, j}, c_{i, j}, d_{i}$, we call (9) is QCT-B spline basis with 2 shape parameters.

For equidistant knots, the QCT-B spline basis is called a uniform QCT-B spline basis, and the corresponding knot vector is called an equidistant knot vector. For non-uniform knots, the QCT-B spline basis is called a non-uniform QCT-B spline basis, and the corresponding knot vector is called a non-equidistant knot vector. Fig.8 shows the image of the uniform QCT-B-spline basis under different shape parameters.
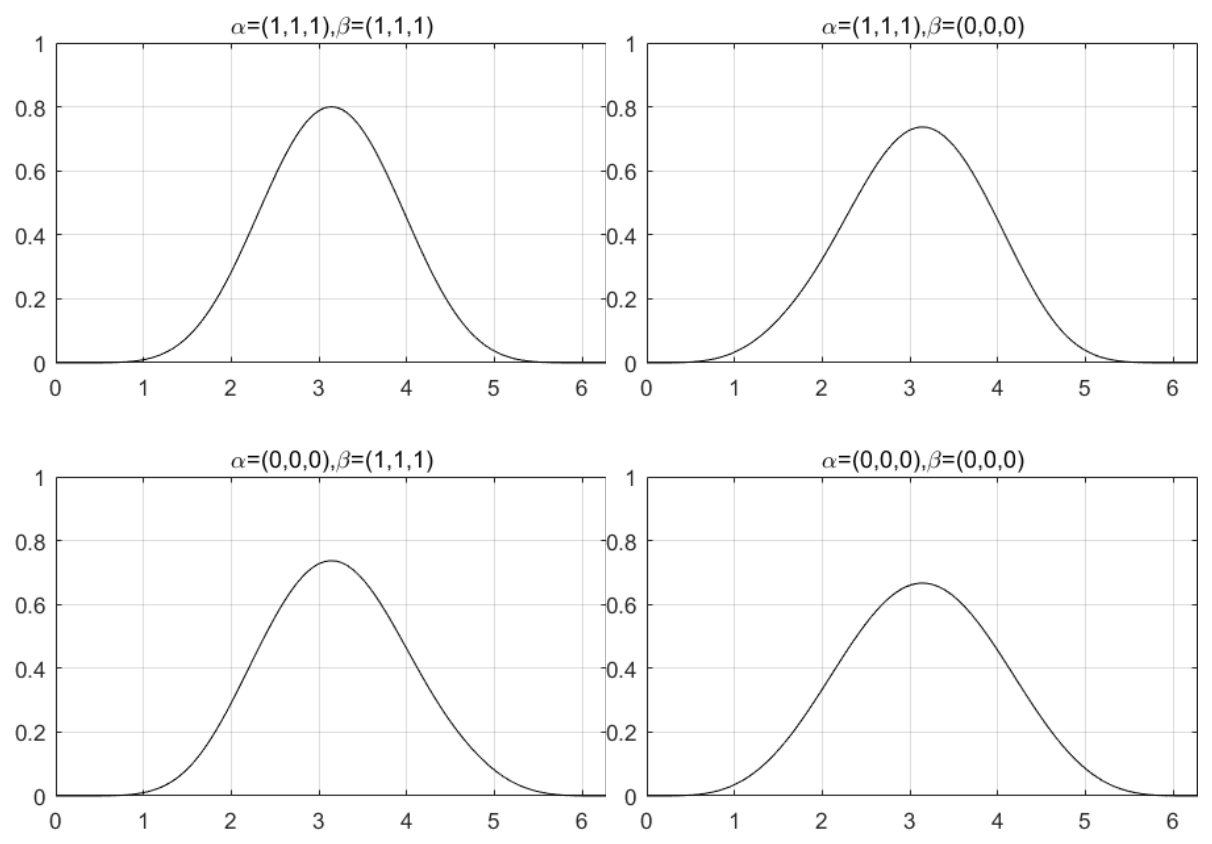

Fig.8 Uniform B-spline basis

Direct calculations yield the following lemma, which will be very useful for subsequent discussions.

Lemma 1 For any $i \in Z$, the following equation is true:

$$
\begin{aligned}
& \text { (1) } a_{i}+b_{i 0}+c_{i 0}=1, \quad \text { (2) } b_{i 1}+c_{i 1}=1, \quad \text { (3) } b_{i 2}+c_{i 2}=1 \text {, } \\
& \text { (4) } b_{i 3}+c_{i 3}+d_{i}=1, \quad \text { (5) } d_{i}=c_{i+1,0} \text {, } \\
& \text { (6) } b_{i+2,0}=c_{i+1,3}, \quad \text { (7) } b_{i+2,3}=a_{i+3} \text {, } \\
& \text { (8) }\left(\frac{\pi}{2 h_{i}}\right)\left(\beta_{i}+3\right) d_{i}=\left(\frac{\pi}{2 h_{i+1}}\right)\left(\alpha_{i+1}+3\right)\left(c_{i+1,1}-c_{i+1,0}\right) \text {, } \\
& \text { (9) }\left(\frac{\pi}{2 h_{i+1}}\right)\left(\beta_{i+1}+3\right)\left(c_{i+1,3}-c_{i+1,2}\right)=\left(\frac{\pi}{2 h_{i+2}}\right)\left(\alpha_{i+2}+3\right)\left(b_{i+2,1}-b_{i+2,0}\right) \text {, }
\end{aligned}
$$




$$
\begin{aligned}
& \text { (10) }\left(\frac{\pi}{2 h_{i+2}}\right)\left(\beta_{i+2}+3\right)\left(b_{i+2,3}-b_{i+2,2}\right)=-\left(\frac{\pi}{2 h_{i+3}}\right)\left(\alpha_{i+3}+3\right) a_{i+3}, \\
& \text { (11) }\left(\frac{\pi}{2 h_{i}}\right)^{2} 6\left(\beta_{i}+1\right) d_{i}=\left(\frac{\pi}{2 h_{i+1}}\right)^{2}\left[\left(6 \alpha_{i+1}+6\right) c_{i+1,0}-\left(6 \alpha_{i+1}+8\right) c_{i+1,1}+2 c_{i+1,2}\right], \\
& \text { (12) }\left(\frac{\pi}{2 h_{i+1}}\right)^{2}\left[2 c_{i+1,1}-c_{i+1,2}\left(6 \beta_{i+1}+8\right)+\left(6 \beta_{i+1}+6\right) c_{i+1,3}\right] \\
& =\left(\frac{\pi}{2 h_{i+2}}\right)^{2}\left[\left(6 \alpha_{i+2}+6\right) b_{i+2,0}-\left(6 \alpha_{i+2}+8\right) b_{i+2,1}+2 b_{i+2,2}\right], \\
& \text { (13) }\left(\frac{\pi}{2 h_{i+2}}\right)^{2}\left[2 b_{i+2,1}-b_{i+2,2}\left(6 \beta_{i+2}+8\right)+b_{i+2,3}\left(6 \beta_{i+2}+6\right)\right]=\left(\frac{\pi}{2 h_{i+3}}\right)^{2}\left(6 \alpha_{i+3}+6\right) a_{i+3},
\end{aligned}
$$

Remark 1 For $u_{i+j}=u_{i}+j h, h>0, j=1,2,3,4$, when $\alpha_{i+1}=\beta_{i}$, by simplifying the basis function (22) from two parameters to one parameter, the corresponding coefficient $a_{i}, b_{i, j}, c_{i, j}, d_{i}$ can be simplified to as follows:

$$
\begin{aligned}
& \lambda_{i}=6\left(\alpha_{i+1}+1\right)\left(\alpha_{i+1}+3\right) \quad \mu_{i}=2\left(\alpha_{i+1}+3\right) \\
& \phi_{i}=\frac{\lambda_{i}+\mu_{i}}{\alpha_{i+1}+3} \quad \varphi_{i}=\frac{\lambda_{i-1}+\mu_{i-1}}{\alpha_{i}+3} \\
& a_{i}=\frac{\left(\alpha_{i}+3\right) \lambda_{i-2}}{\lambda_{i-1} \mu_{i-2}+\lambda_{i-2} \lambda_{i-1}+\lambda_{i-2} \mu_{i-1}} \\
& d_{i}=\frac{\left(\alpha_{i+1}+3\right) \lambda_{i+1}}{\lambda_{i+1} \mu_{i}+\lambda_{i} \lambda_{i+1}+\lambda_{i} \mu_{i+1}} \\
& b_{i, 0}=\frac{\left(\alpha_{i}+3\right) \phi_{i}}{\mu_{i-1}} a_{i+1}+\frac{\left(\alpha_{i}+3\right) \varphi_{i-1}}{\mu_{i-1}} d_{i-2} \\
& b_{i, 1}=\phi_{i} a_{i+1} \quad b_{i, 2}=\frac{\mu_{i}}{\alpha_{i+1}+3} a_{i+1} \quad b_{i, 3}=a_{i+1} \\
& c_{i, 0}=d_{i-1} \quad c_{i, 1}=\frac{\mu_{i-1}}{\alpha_{i}+3} d_{i-1} \quad c_{i, 2}=\varphi_{i} d_{i-1} \\
& c_{i, 3}=\frac{\left(\alpha_{i+1}+3\right) \phi_{i+1}}{\mu_{i}} a_{i+2}+\frac{\left(\alpha_{i+1}+3\right) \varphi_{i}}{\mu_{i}} d_{i-1}
\end{aligned}
$$

When $\alpha=\beta=0$, we have

$$
\begin{aligned}
& d_{i}=\frac{1}{10}, \quad c_{i+1,0}=\frac{1}{5}, \quad c_{i+1,1}=\frac{1}{5}, \quad c_{i+1,2}=\frac{4}{5}, \quad c_{i+1,3}=\frac{4}{5}, \\
& b_{i+1,0}=\frac{4}{5}, \quad b_{i+1,1}=\frac{4}{5}, \quad b_{i+1,2}=\frac{1}{5}, \quad b_{i+1,3}=\frac{1}{5}, \quad a_{i+3}=\frac{1}{10} \text {, }
\end{aligned}
$$

\subsection{Properties of the non-uniform QCT-B spline basis}

Theorem 5 For any $u \in\left[u_{3}, u_{n+1}\right], \sum_{i=0}^{n} B_{i}(u)=1$.

Proof For $u \in\left[u_{i}, u_{i+1}\right), i=3,4, \cdots, n$, we have $B_{j}(u)=0, j \neq i-3, i-2, i-1, i$.

$$
\begin{array}{ll}
B_{i-3}(u)=a_{i} A_{0}\left(t_{i} ; \alpha_{i}\right), & B_{i-2}(u)=\sum_{j=0}^{3} b_{i, j} A_{j}\left(t_{i} ; \alpha_{i}, \beta_{i}\right), \\
B_{i-1}(u)=\sum_{j=0}^{3} c_{i, j} A_{j}\left(t_{i} ; \alpha_{i}, \beta_{i}\right), & B_{i}(u)=d_{i} A_{3}\left(t_{i}, \beta_{i}\right),
\end{array}
$$

By using Lemma1, we have

$$
\sum_{i=0}^{n} B_{i}(u)=a_{i} A_{0}\left(t_{i} ; \alpha_{i}\right)+\sum_{j=0}^{3} b_{i, j} A_{j}\left(t_{i} ; \alpha_{i}, \beta_{i}\right)+\sum_{j=0}^{3} c_{i, j} A_{j}\left(t_{i} ; \alpha_{i}, \beta_{i}\right)+d_{i} A_{3}\left(t_{i} ; \beta_{i}\right)=\sum_{j=0}^{3} A_{j}\left(t_{i} ; \beta_{i}\right)=1 .
$$

Theorem 6 If $\alpha_{i}, \beta_{i} \in[-1,1]$, for any $u_{i}<u<u_{i+4}$, we have $B_{i}(u)>0$.

Proof For $\alpha_{i}, \beta_{i} \in[-1,1]$, we have $a_{i}, b_{i, j}, c_{i, j}, d_{i}>0$. According to the nonnegative of $A_{j}\left(t_{i} ; \alpha_{i}, \beta_{i}\right)$, $j=0,1,2,3$, we can easily get $B_{i}(u)>0$. 
Theorem 7 For any $\alpha_{i}, \beta_{i} \in[-1,1],\left\{B_{0}(u), B_{1}(u), \cdots, B_{n}(u)\right\}$ are linear independent on $\left[u_{3}, u_{n+1}\right]$.

Proof For any $\xi_{i} \in R(i=0,1, \cdots, n), u \in\left[u_{3}, u_{n+1}\right]$, we consider

$$
B(u)=\sum_{i=0}^{n} \xi_{i} B_{i}(u)=0 .
$$

For $\alpha_{i}, \beta_{i} \in[-1,1]$, we have

$$
\begin{aligned}
& B\left(u_{i}\right)=a_{i} \xi_{i-3}+b_{i, 0} \xi_{i-2}+c_{i, 0} \xi_{i-1}=0, \\
& B^{\prime}\left(u_{i}\right)=\frac{\pi}{2 h_{i}}\left[\left(\alpha_{i}+3\right) a_{i}\left(\xi_{i-2}-\xi_{i-3}\right)+\frac{\left(\beta_{i-1}+3\right) d_{i-1} h_{i}}{h_{i-1}}\left(\xi_{i-1}-\xi_{i-2}\right)\right]=0, \\
& B^{\prime \prime}\left(u_{i}\right)=\left(\frac{\pi}{2 h_{i}}\right)^{2}\left[6\left(\alpha_{i}+1\right) a_{i}\left(\xi_{i-3}-\xi_{i-2}\right)+\frac{6\left(\beta_{i-1}+1\right) d_{i-1} h_{i}^{2}}{h_{i-1}^{2}}\left(\xi_{i-1}-\xi_{i-2}\right)\right]=0 .
\end{aligned}
$$

Thus, we have

$$
\left\{\begin{array}{l}
a_{i}\left(\xi_{i-3}-\xi_{i-2}\right)+\left(a_{i}+b_{i, 0}+c_{i, 0}\right) \xi_{i-2}+c_{i, 0}\left(\xi_{i-1}-\xi_{i-2}\right)=0 \\
-\left(\alpha_{i}+3\right) a_{i}\left(\xi_{i-3}-\xi_{i-2}\right)+\frac{\left(\beta_{i-1}+3\right) d_{i-1} h_{i}}{h_{i-1}}\left(\xi_{i-1}-\xi_{i-2}\right)=0 \\
\left(\alpha_{i}+1\right) a_{i}\left(\xi_{i-3}-\xi_{i-2}\right)+\frac{\left(\beta_{i-1}+1\right) d_{i-1} h_{i}^{2}}{h_{i-1}^{2}}\left(\xi_{i-1}-\xi_{i-2}\right)=0
\end{array}\right.
$$

According to $a_{i}+b_{i 0}+c_{i 0}=1$, the related coefficient determinant is

$$
\left|M_{i}\right|=\left|\begin{array}{ccc}
a_{i} & 1 & c_{i, 0} \\
-\left(\alpha_{i}+3\right) a_{i} & 0 & \frac{\left(\beta_{i-1}+3\right) d_{i-1} h_{i}}{h_{i-1}} \\
\left(\alpha_{i}+1\right) a_{i} & 0 & \frac{\left(\beta_{i-1}+1\right) d_{i-1} h_{i}^{2}}{h_{i-1}^{2}}
\end{array}\right|=\frac{a_{i} d_{i-1} h_{i}}{h_{i-1}^{2}}\left[\left(\beta_{i-1}+1\right) h_{i}\left(\alpha_{i}+3\right)+\left(\alpha_{i}+1\right)\left(\beta_{i-1}+3\right) h_{i-1}\right]>0 .
$$

Therefore, we can get $\xi_{i-3}=\xi_{i-2}=\xi_{i-1}=0, i=3,4, \ldots, n+1$.

Theorem 8 For any $u \in\left[u_{i}, u_{i+1}\right], \alpha_{i}, \beta_{i} \in[-1,1], i=3,4, \ldots, n$, the systems $\left(B_{i-3}(u), B_{i-2}(u), B_{i-1}(u), B_{i}(u)\right)$ is a totally positive basis of the space $T_{\alpha_{i}, \beta_{i}}$.

Proof For $u \in\left[u_{i}, u_{i+1}\right], i=3,4, \ldots, n$, we have

$$
\left(B_{i-3}(u), B_{i-2}(u), B_{i-1}(u), B_{i}(u)\right)=\left(T_{0}\left(t_{i} ; \alpha_{i}\right), T_{1}\left(t_{i} ; \alpha_{i}\right), T_{2}\left(t_{i} ; \beta_{i}\right), T_{3}\left(t_{i} ; \beta_{i}\right)\right) H_{i}
$$

where,

$$
H_{i}=\left[\begin{array}{cccc}
a_{i} & b_{i, 0} & c_{i, 0} & 0 \\
0 & b_{i, 1} & c_{i, 1} & 0 \\
0 & b_{i, 2} & c_{i, 2} & 0 \\
0 & b_{i, 3} & c_{i, 3} & d_{i}
\end{array}\right],
$$

and $\alpha_{i}, \beta_{i} \in[-1,1], t_{i}(u)=\pi\left(u-u_{i}\right) / 2 h_{i}$. Given that the system $\left(T_{0}\left(t_{i} ; \alpha_{i}\right), T_{1}\left(t_{i} ; \alpha_{i}\right), T_{2}\left(t_{i} ; \beta_{i}\right), T_{3}\left(t_{i} ; \beta_{i}\right)\right)$ is a $\mathrm{B}$ basis of the space $T_{\alpha_{i}, \beta_{i}}$. According to Theorem 4.2 in [31], we can conclude that $H_{i}$ is a nonsingular stochastic and totally positive matrix.

For $\alpha_{i}, \beta_{i} \in[-1,1]$, we have $a_{i}, b_{i, j}, c_{i, j}, d_{i}>0$. Moreover, it is easily obtain that $H_{i}$ is a nonsingular stochastic matrix from Lemma 1. Straightforward computation provides

$$
\begin{aligned}
& \left|\begin{array}{ll}
b_{i, 0} & c_{i, 0} \\
b_{i, 1} & c_{i, 1}
\end{array}\right|=\frac{\left(\beta_{i-1}+3\right) \varphi_{i-1} h_{i}}{\left(\alpha_{i}+3\right) h_{i-1}} d_{i-2} d_{i-1}>0, \\
& \left|\begin{array}{ll}
b_{i, 0} & c_{i, 0} \\
b_{i, 2} & c_{i, 2}
\end{array}\right|=\frac{h_{i}\left(\lambda_{i-1} \mu_{i} h_{i+1}+\lambda_{i-1} \lambda_{i} h_{i}+\lambda_{i} \mu_{i-1} h_{i-1}\right)}{\left(\beta_{i}+3\right) \mu_{i-1} h_{i-1} h_{i+1}^{2}}\left(a_{i+1}+3\right) d_{i-1}+\frac{\left(\beta_{i-1}+3\right) \varphi_{i-1} \varphi_{i} h_{i}}{\mu_{i-1}} d_{i-2} d_{i-1}>0,
\end{aligned}
$$




$$
\begin{aligned}
\left|\begin{array}{ll}
b_{i, 0} & c_{i, 0} \\
b_{i, 3} & c_{i, 3}
\end{array}\right| & =\frac{\left(\alpha_{i}+3\right)\left(\alpha_{i+1}+3\right) \phi_{i} \phi_{i+1} h_{i-1} h_{i}}{\mu_{i-1} \mu_{i}} a_{i+1} a_{i+2}+\frac{h_{i}\left(\lambda_{i-1} \mu_{i} h_{i+1}+\lambda_{i-1} \lambda_{i} h_{i}+\lambda_{i} \mu_{i-1} h_{i-1}\right)}{\mu_{i-1} \mu_{i} h_{i-1} h_{i+1}} a_{i+1} d_{i-1} \\
& +\frac{\left(\alpha_{i+1}+3\right)\left(\beta_{i-1}+3\right) \phi_{i+1} \varphi_{i-1} h_{i}^{2}}{\mu_{i-1} \mu_{i}} a_{i+2} d_{i-2}+\frac{\left(\beta_{i-1}+3\right)\left(\beta_{i}+3\right) \varphi_{i-1} \varphi_{i} h_{i} h_{i+1}}{\mu_{i-1} \mu_{i}} d_{i-2} d_{i-1}>0, \\
\left|\begin{array}{ll}
b_{i, 1} & c_{i, 1} \\
b_{i, 2} & c_{i, 2}
\end{array}\right| & =\frac{h_{i}\left(\lambda_{i-1} \mu_{i} h_{i+1}+\lambda_{i-1} \lambda_{i} h_{i}+\lambda_{i} \mu_{i-1} h_{i-1}\right)}{\left(\alpha_{i}+3\right)\left(\beta_{i}+3\right) \mu_{i-1} h_{i-1}^{2} h_{i+1}^{2}} a_{i+1} d_{i-1}>0, \\
\left|\begin{array}{ll}
b_{i, 1} & c_{i, 1} \\
b_{i, 3} & c_{i, 3}
\end{array}\right| & =\frac{h_{i}\left(\lambda_{i-1} \mu_{i} h_{i+1}+\lambda_{i-1} \lambda_{i} h_{i}+\lambda_{i} \mu_{i-1} h_{i-1}\right)}{\left(\alpha_{i}+3\right) \mu_{i} h_{i-1}^{2} h_{i+1}} a_{i+1} d_{i-1}+\frac{\left(\alpha_{i+1}+3\right) \phi_{i} \phi_{i+1} h_{i}}{\mu_{i}} a_{i+1} a_{i+2}>0, \\
\left|\begin{array}{ll}
b_{i, 2} & c_{i, 2} \\
b_{i, 3} & c_{i, 3}
\end{array}\right| & =\frac{\left(\alpha_{i+1}+3\right) \phi_{i+1} h_{i}}{\left(\beta_{i}+3\right) h_{i+1}} a_{i+1} a_{i+2}>0,
\end{aligned}
$$

Thus, we can easily proof that $H_{i}$ is a totally positive matrix. The corresponding conclusions can be proved.

Theorem 9 For any non-uniform knot vector $U$, when $\alpha_{i}, \beta_{i} \in[-1,1], B_{i}(u)$ has $C^{2}$ continuity at each knot.

Proof We only consider the situation at knot $u_{i+1}$. For any $\alpha_{i}, \beta_{i} \in[-1,1]$, we have

$$
\begin{aligned}
& B_{i}\left(u_{i+1}^{-}\right)=d_{i}, \quad B_{i}\left(u_{i+1}^{+}\right)=c_{i+1,0}, \\
& B_{i}^{\prime}\left(u_{i+1}^{-}\right)=\left(\frac{\pi}{2 h_{i}}\right)\left(\beta_{i}+3\right) d_{i}, \quad B_{i}^{\prime}\left(u_{i+1}^{+}\right)=\left(\frac{\pi}{2 h_{i+1}}\right)\left(\alpha_{i+1}+3\right)\left(c_{i+1,1}-c_{i+1,0}\right), \\
& B_{i}^{\prime \prime}\left(u_{i+1}^{-}\right)=\left(\frac{\pi}{2 h_{i}}\right)^{2} 6\left(\beta_{i}+1\right) d_{i}, \quad B_{i}^{\prime \prime}\left(u_{i+1}^{+}\right)=\left(\frac{\pi}{2 h_{i+1}}\right)^{2}\left[\left(6 \alpha_{i+1}+6\right) c_{i+1,0}-\left(6 \alpha_{i+1}+8\right) c_{i+1,1}+2 c_{i+1,2}\right] .
\end{aligned}
$$

Thus, by simple computing the theorem is proved at knot $u_{i+1}$. Other knots can be verified in the same way.

Theorem 10 For any uniform knot vector $U$, and $\alpha_{i+1}=\beta_{i} \in[-1,1], \quad B_{i}(u)$ has $C^{2 n-1}(n=1,2,3, \cdots)$ continuity at each knot.

Proof First, we use verify that the $(2 n-1)$-order derivative of (3) has the following form:

$$
\left\{\begin{array}{l}
T_{0}^{(2 n-1)}=(-1)^{n} \frac{1}{4}\left[(13 \alpha+15) \cos t-3^{2 n-1}(3 \alpha+1) \cos 3 t-4^{n}(4 \alpha+3) \sin 2 t+2 \alpha \cdot 16^{n-1} \sin 4 t\right] \\
T_{1}^{(2 n-1)}=-(-4)^{n-1} \sin 2 t-(-1)^{n} \frac{1}{4}\left[(13 \alpha+15) \cos t-3^{2 n-1}(3 \alpha+1) \cos 3 t-4^{n}(4 \alpha+3) \sin 2 t+2 \alpha \cdot 16^{n-1} \sin 4 t\right] \\
T_{2}^{(2 n-1)}=(-4)^{n-1} \sin 2 t-(-1)^{n-1} \frac{1}{4}\left[(13 \beta+15) \sin t+3^{2 n-1}(3 \beta+1) \sin 3 t-4^{n}(4 \beta+3) \sin 2 t-2 \beta \cdot 16^{n-1} \sin 4 t\right] \\
T_{3}^{(2 n-1)}=(-1)^{n-1} \frac{1}{4}\left[(13 \beta+15) \sin t+3^{2 n-1}(3 \beta+1) \sin 3 t-4^{n}(4 \beta+3) \sin 2 t-2 \beta \cdot 16^{n-1} \sin 4 t\right]
\end{array}\right.
$$

From the the theory of mathematical induction, we have

(1) When $n=1$, we can conclude that

$$
\left\{\begin{array}{l}
A_{0}^{\prime}=-\frac{1}{4}[(13 \alpha+15) \cos t-3(3 \alpha+1) \cos 3 t-4(4 \alpha+3) \sin 2 t+2 \alpha \sin 4 t] \\
A_{1}^{\prime}=-\sin 2 t+\frac{1}{4}[(13 \alpha+15) \cos t-3(3 \alpha+1) \cos 3 t-4(4 \alpha+3) \sin 2 t+2 \alpha \sin 4 t] \\
A_{2}^{\prime}=\sin 2 t-\frac{1}{4}[(13 \beta+15) \sin t+3(3 \beta+1) \sin 3 t-4(4 \beta+3) \sin 2 t-2 \beta \sin 4 t] \\
A_{3}^{\prime}=\frac{1}{4}[(13 \beta+15) \sin t+3(3 \beta+1) \sin 3 t-4(4 \beta+3) \sin 2 t-2 \beta \sin 4 t]
\end{array}\right.
$$

Thus, The form (23) is meet when $n=1$.

We assume that the form (23) is also meet when $n=k$. Therefore, the $(2 n-1)$-order derivative of (3) are 


$$
\left\{\begin{array}{l}
A_{0}^{(2 k-1)}=(-1)^{k} \frac{1}{4}\left[(13 \alpha+15) \cos t-3^{2 k-1}(3 \alpha+1) \cos 3 t-4^{k}(4 \alpha+3) \sin 2 t+2 \alpha \cdot 16^{k-1} \sin 4 t\right] \\
A_{1}^{(2 k-1)}=-(-4)^{k-1} \sin 2 t-(-1)^{k} \frac{1}{4}\left[(13 \alpha+15) \cos t-3^{2 k-1}(3 \alpha+1) \cos 3 t-4^{k}(4 \alpha+3) \sin 2 t+2 \alpha \cdot 16^{k-1} \sin 4 t\right] \\
A_{2}^{(2 k-1)}=(-4)^{k-1} \sin 2 t-(-1)^{k-1} \frac{1}{4}\left[(13 \beta+15) \sin t+3^{2 k-1}(3 \beta+1) \sin 3 t-4^{k}(4 \beta+3) \sin 2 t-2 \beta \cdot 16^{k-1} \sin 4 t\right] \\
A_{3}^{(2 k-1)}=(-1)^{k-1} \frac{1}{4}\left[(13 \beta+15) \sin t+3^{2 k-1}(3 \beta+1) \sin 3 t-4^{k}(4 \beta+3) \sin 2 t-2 \beta \cdot 16^{k-1} \sin 4 t\right]
\end{array}\right.
$$

Thus, we have

$\left\{\begin{array}{l}{\left[A_{0}^{(2 k-1)}\right]^{\prime \prime}=(-1)^{k+1} \frac{1}{4}\left[(13 \alpha+15) \cos t-3^{2(k+1)-1}(3 \alpha+1) \cos 3 t-4^{k+1}(4 \alpha+3) \sin 2 t+2 \alpha \cdot 16^{k+1-1} \sin 4 t\right]=A_{0}^{(2(k+1)-1)}} \\ {\left[A_{1}^{(2 k-1)}\right]^{\prime \prime}=-(-4)^{k+1-1} \sin 2 t-(-1)^{k+1} \frac{1}{4}\left[(13 \alpha+15) \cos t-3^{2(k+1)-1}(3 \alpha+1) \cos 3 t-4^{k+1}(4 \alpha+3) \sin 2 t+2 \alpha \cdot 16^{k+1-1} \sin 4 t\right]=A_{1}^{(2(k+1)-1)}} \\ {\left[A_{2}^{(2 k-1)}\right]^{\prime \prime}=(-4)^{k+1-1} \sin 2 t-(-1)^{k+1-1} \frac{1}{4}\left[(13 \beta+15) \sin t+3^{2(k+1)-1}(3 \beta+1) \sin 3 t-4^{k+1}(4 \beta+3) \sin 2 t-2 \beta \cdot 16^{k+1-1} \sin 4 t\right]=A_{2}^{(2(k+1)-1)}} \\ {\left[A_{3}^{(2 k-1)}\right]^{\prime \prime}=(-1)^{k+1-1} \frac{1}{4}\left[(13 \beta+15) \sin t+3^{2(k+1)-1}(3 \beta+1) \sin 3 t-4^{k+1}(4 \beta+3) \sin 2 t-2 \beta \cdot 16^{k+1-1} \sin 4 t\right]=A_{3}^{(2(k+1)-1)}}\end{array}\right.$

We can easily find that the form (23) is meet when $n=k+1$ as well. In summary, the $(2 n-1)$-order derivative of (3) meet the form of (23). Then, we given the proof that $B_{i}(u)$ is $C^{2 n-1}(n=1,2, \cdots)$ continuous at each knot.

We only consider the continuity at the knot $u_{i+1}$. From here and Remark 1, for uniform knot and the condition of $\alpha_{i+1}=\beta_{i} \in[-1,1]$, we can get $d_{i}=c_{i+1,1}-c_{i+1,0}$. According to the expression of (22), we have

$$
\begin{aligned}
B_{i}^{(2 n-1)}\left(u_{i+1}^{-}\right) & =d_{i} A_{3}^{(2 n-1)}\left(\pi / 2 ; \beta_{i}\right)=(-1)^{n-1} \frac{1}{4}\left[\left(13 \beta_{i}+15\right)-3^{2 n-1}\left(3 \beta_{i}+1\right)\right] d_{i}, \\
B_{i}^{(2 n-1)}\left(u_{i+1}^{+}\right) & =c_{i+1,0} A_{0}^{(2 n-1)}\left(0 ; \alpha_{i+1,} \beta_{i}\right)+c_{i+1,1} A_{1}^{(2 n-1)}\left(0 ; \alpha_{i+1,} \beta_{i}\right)+c_{i+1,2} A_{2}^{(2 n-1)}\left(0 ; \alpha_{i+1,} \beta_{i}\right)+c_{i+1,3} A_{3}^{(2 n-1)}\left(0 ; \alpha_{i+1,} \beta_{i}\right) \\
= & (-1)^{n} \frac{1}{4}\left[\left(7 \alpha_{i+1}+8\right)-3^{2 n-1}\left(3 \alpha_{i+1}+1\right)\right]\left(c_{i+1,0}-c_{i+1,1}\right),
\end{aligned}
$$

Based on the above conditions, we have $B_{i}^{(2 n-1)}\left(u_{i+1}^{-}\right)=B_{i}^{(2 n-1)}\left(u_{i+1}^{+}\right)$. It can therefore be proved that $B_{i}(u)$ has $C^{2 n-1}(n=1,2, \cdots)$ continuity at the knot $u_{i+1}$. The basis function $B_{i}(u)$ can be similarly discussed with respect to other knot continuity.

\section{The Non-uniform QCT-spline curves}

\subsection{The definition and properties of curves}

Definition 6 For any non-uniform knot vector $U, P_{i}(i=0,1, \ldots, n) \in R^{2} / R^{3}, n \geq 3$, and $\alpha_{i}, \beta_{i} \in[-1,1], n \geq 3, u \in\left[u_{3}, u_{n+1}\right]$, we call

$$
Q(u)=\sum_{i=0}^{n} B_{i}(u) P_{i}
$$

as a non-uniform QCT-B spline curve with two shape parameters.

Obviously, for $u \in\left[u_{i}, u_{i+1}\right], i=3,4, \ldots, n$, the QCT-B spline curve segment curve can be expressed as following form

$$
\begin{aligned}
& Q_{i}(u)=\sum_{j=i-3}^{i} B_{j}(u) P_{j}=\left(a_{i} P_{i-3}+b_{i 0} P_{i-2}+c_{i 0} P_{i-1}\right) T_{0}\left(t_{i} ; \alpha_{i}\right)+ \\
& \quad\left(b_{i 1} P_{i-2}+c_{i 1} P_{i-1}\right) T_{1}\left(t_{i} ; \alpha_{i}\right)+\left(b_{i 2} P_{i-2}+c_{i 2} P_{i-1}\right) T_{2}\left(t_{i} ; \beta_{i}\right)+\left(b_{i 3} P_{i-2}+c_{i 3} P_{i-1}+d_{i} P_{i}\right) T_{3}\left(t_{i} ; \beta_{i}\right) .
\end{aligned}
$$

Available from Theorem 5 and 6 , for $u \in\left[u_{i}, u_{i+1}\right], i=3,4, \ldots, n$, the non-uniform QCT-B spline curve $Q_{i}(u)$ possesses affine invariance and it is located within the convex formed by the control points $P_{i-3}, P_{i-2}, P_{i-1}, P_{i}$. Furthermore, it can be see from Theorem 8 that the non-uniform QCT-B spline curve has variation diminishing. Therefore, the non-uniform QCT-B spline curve retains all the basic properties of the traditional B-spline and has flexible shape adjustability for geometric design. The following theorem can be obtained from Theorem 9 and 10 .

Theorem 11 For any non-uniform knot vector $U$, when $\alpha_{i}, \beta_{i} \in[-1,1]$, the non-uniform QCT-B spline 
curve is $C^{2}$ continuity at each knot.

Proof We only consider the continuity of the non-uniform QCT-B spline curve at the knot $u_{i+1}$. Then from equation (26), in the interval $\left[u_{i}, u_{i+1}\right]$ and $\left[u_{i+1}, u_{i+2}\right]$, the non-uniform QCT-B spline curve segments can be expressed as

$$
\begin{gathered}
Q_{i}(u)=\sum_{j=i-3}^{i} B_{j}(u) P_{j}=\left(a_{i} P_{i-3}+b_{i 0} P_{i-2}+c_{i 0} P_{i-1}\right) T_{0}\left(t_{i} ; \alpha_{i}\right)+\left(b_{i 1} P_{i-2}+c_{i 1} P_{i-1}\right) T_{1}\left(t_{i} ; \alpha_{i}\right)+ \\
\left(b_{i 2} P_{i-2}+c_{i 2} P_{i-1}\right) T_{2}\left(t_{i} ; \beta_{i}\right)+\left(b_{i 3} P_{i-2}+c_{i 3} P_{i-1}+d_{i} P_{i}\right) T_{3}\left(t_{i} ; \beta_{i}\right), u \in\left[u_{i}, u_{i+1}\right] . \\
Q_{i+1}(u)=\sum_{j=i-2}^{i+1} B_{j}(u) P_{j}=\left(a_{i+1} P_{i-2}+b_{i+1,0} P_{i-1}+c_{i+1,0} P_{i}\right) T_{0}\left(t_{i+1} ; \alpha_{i+1}\right)+\left(b_{i+1,1} P_{i-1}+c_{i+1,1} P_{i}\right) T_{1}\left(t_{i+1} ; \alpha_{i+1}\right)+ \\
\left(b_{i+1,2} P_{i-1}+c_{i+1,2} P_{i}\right) T_{2}\left(t_{i+1} ; \beta_{i+1}\right)+\left(b_{i+1,3} P_{i-1}+c_{i+1,3} P_{i}+d_{i+1} P_{i+1}\right) T_{3}\left(t_{i+1} ; \beta_{i+1}\right), u \in\left[u_{i+1}, u_{i+2}\right] .
\end{gathered}
$$

Thus, we have

$$
\begin{aligned}
& Q_{i}\left(u_{i+1}^{+}\right)=b_{i 3} P_{i-2}+c_{i 3} P_{i-1}+d_{i} P_{i}, \\
& Q_{i+1}\left(u_{i+1}^{-}\right)=a_{i+1} P_{i-2}+b_{i+1,0} P_{i-1}+c_{i+1,0} P_{i}, \\
& Q_{i}^{\prime}\left(u_{i+1}^{+}\right)=-\left(b_{i 2} P_{i-2}+c_{i 2} P_{i-1}\right)\left(\beta_{i}+3\right)+\left(b_{i 3} P_{i-2}+c_{i 3} P_{i-1}+d_{i} P_{i}\right)\left(\beta_{i}+3\right), \\
& Q_{i+1}^{\prime}\left(u_{i+1}^{-}\right)=-\left(a_{i+1} P_{i-2}+b_{i+1,0} P_{i-1}+c_{i+1,0} P_{i}\right)\left(3+\alpha_{i+1}\right)+\left(b_{i+1,1} P_{i-1}+c_{i+1,1} P_{i}\right)\left(3+\alpha_{i+1}\right), \\
& Q_{i}^{\prime \prime}\left(u_{i+1}^{+}\right)=2\left(b_{i 1} P_{i-2}+c_{i 1} P_{i-1}\right)+\left(b_{i 2} P_{i-2}+c_{i 2} P_{i-1}\right)\left[-2-\left(6 \beta_{i}+6\right)\right]+\left(b_{i 3} P_{i-2}+c_{i 3} P_{i-1}+d_{i} P_{i}\right)\left(6 \beta_{i}+6\right), \\
& Q_{i+1}^{\prime \prime}\left(u_{i+1}^{-}\right)=\left(a_{i+1} P_{i-2}+b_{i+1,0} P_{i-1}+c_{i+1,0} P_{i}\right)\left(6 \alpha_{i+1}+6\right)+\left(b_{i+1,1} P_{i-1}+c_{i+1,1} P_{i}\right)\left[-2-\left(6 \alpha_{i+1}+6\right)\right]+2\left(b_{i+1,2} P_{i-1}+c_{i+1,2} P_{i}\right) .
\end{aligned}
$$

According to the Theorem 9 and the coefficients $a_{i}, b_{i, j}, c_{i, j}, d_{i}$ of expression (22), we have $Q_{i}\left(u_{i+1}^{+}\right)=$ $Q_{i+1}\left(u_{i+1}^{-}\right), Q_{i}^{\prime}\left(u_{i+1}^{+}\right)=Q_{i+1}^{\prime}\left(u_{i+1}^{-}\right), Q_{i}^{\prime \prime}\left(u_{i+1}^{+}\right)=Q_{i+1}^{\prime \prime}\left(u_{i+1}^{-}\right)$.

Theorem 12 For any uniform knot vector, for $\alpha_{i}, \beta_{i} \in[-1,1]$ and $\alpha_{i+1}=\beta_{i}, B(u)$ has $C^{2 n-1}(n=1,2,3, \cdots)$ continuity at each knot.

Proof We only consider the continuity of the uniform QCT-B spline curve at the knot $u_{i+1}$. From the Theorem 8 and Theorem 9, we have

$$
\begin{aligned}
& Q_{i}\left(u_{i+1}^{-}\right)=\sum_{j=i-3}^{i} B_{j}\left(u_{i+1}^{-}\right) P_{j} \\
& Q_{i+1}\left(u_{i+1}^{+}\right)=\sum_{j=i-2}^{i+1} B_{j}\left(u_{i+1}^{+}\right) P_{j}
\end{aligned}
$$

We also have $B_{i-3}\left(u_{i+1}^{-}\right)=B_{i+1}\left(u_{i+1}^{+}\right)=0$, so we can easily obtain $Q_{i}\left(u_{i+1}^{-}\right)=Q_{i+1}\left(u_{i+1}^{+}\right)$.
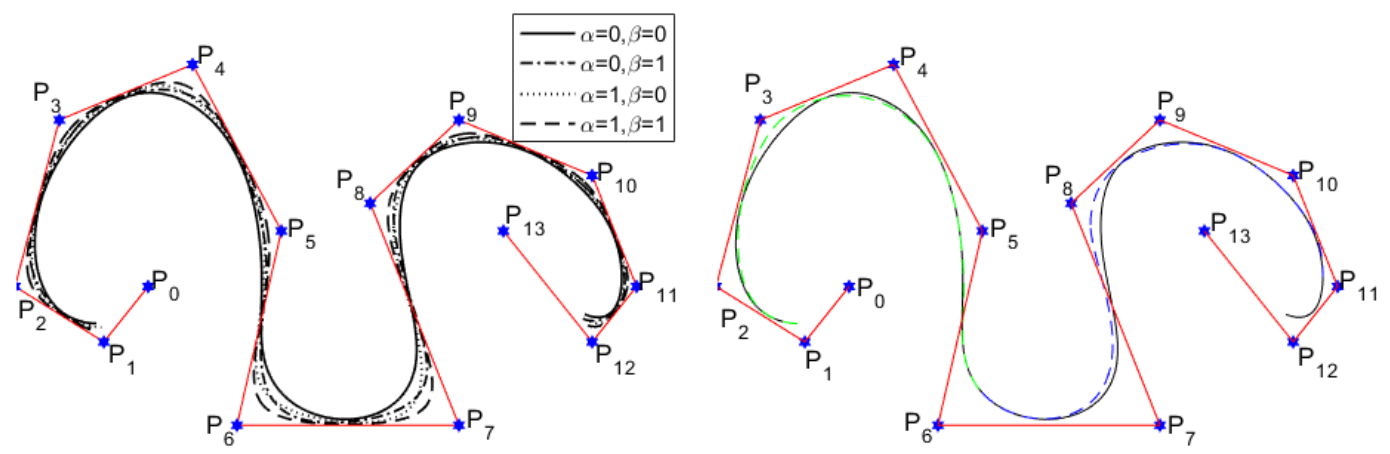

Fig.9 The QCT-B spline curve

\subsection{Local adjustable properties}

The non-uniform QCT-B spline curve presented in the paper possesses two shape parameters $\alpha_{i}, \beta_{i}$. Thus, we can easily adjust the proposed curve shape by changing the parameters $\alpha_{i}, \beta_{i}$ when the control points are fixed. From (26), we can get that $\alpha_{i-1}, \alpha_{i}, \alpha_{i+1}, \alpha_{i+2}, \beta_{i-2}, \beta_{i-1}, \beta_{i}, \beta_{i+1}$ affect the curve segment $Q(u), u \in\left[u_{i}, u_{i+1}\right]$. Thus, $\alpha_{i}$ affect four curve segment $\left[u_{i-2}, u_{i+2}\right]$, and $\beta_{i}$ affect four curve segment $\left[u_{i-1}, u_{i+3}\right]$. Furthermore, the 
shape of the proposed curve can be predicted from the shape parameters $\alpha_{i}, \beta_{i}$. As $\alpha_{i}$ and $\beta_{i}$ increase, the coefficients of control points $P_{i-3}$ and $P_{i}$ decrease while the coefficients of $P_{i-2}$ and $P_{i-1}$ increase. Therefore, as $\alpha_{i}$ and $\beta_{i}$ increase at the same time, $Q_{i}(u)\left(u \in\left[u_{i}, u_{i+1}\right]\right)$ tend to the $P_{i-2} P_{i-1}$. If $\alpha_{i}$ and $\beta_{i}$ increase, $Q_{i}(u)\left(u \in\left[u_{i}, u_{i+1}\right]\right)$ will tend to control point $P_{i-2}$ and $P_{i-1}$, respectively.

Fig.9 shows the uniform QCT-B spline curve $Q(u)$. The left figure shows the situation when all parameters $\alpha_{i}(i=3,4, \cdots, n)$ and $\beta_{i}(i=3,4, \cdots, n)$ are equal. The solid black line of the figure shows the case where all parameters are $\alpha_{i}=\beta_{i}=0$, the green dotted line shows the case where one of the parameters is adjusted to $\alpha_{i}=1$, and the blue dotted line shows the case where one of the parameters is adjusted to $\beta_{i}=1$.

\subsection{Shape analysis of QCT-B spline curves}

In this section, we will use the envelope theory and topological mapping theory to describe the shape features of the non-uniform QCT-B spline curve segments given in (26).

6.3.1 Shape analysis of spatial QCT-B spline curves

Theorem 13 When $\alpha_{i}, \beta_{i} \in[-1,1], u \in\left[u_{i}, u_{i+1}\right], i=3,4, \cdots, n$, if the four control points $P_{j}(j=i-3, i-2, i-1, i)$ are not coplanar, the QCT-Bézier curve segments are spatial curve, it does not have cusps, loops and inflection points, and it has the same rotation direction as the control points.

Proof Let $b_{j}=P_{j}-P_{j-1}(j=i-2, i-1, i)$, the QCT-Bézier curve segments given in (26) can be rewrite as

$$
Q_{i}(u)=P_{i-3}+\left[1-B_{i-3}(u)\right] b_{i-2}+\left[B_{i-1}(u)+B_{i}(u)\right] b_{i-1}+B_{i}(u) b_{i} .
$$

Thus, we have $Q_{i}^{\prime}(u)=-B_{i-3}^{\prime}(u) b_{i-2}+\left[B_{i-1}^{\prime}(u)+B_{i}^{\prime}(u)\right] b_{i-1}+B_{i}^{\prime}(u) b_{i}$. Since the four control points $P_{j}(j=i-3, i-2, i-1, i)$ are not coplanar, the knot vector $b_{j}(j=i-2, i-1, i)$ are linear independent, and $Q_{i}^{\prime}(u)=0\left(u_{i}<u<u_{i+1}\right)$. Therefore, we can easily get $B_{i-3}^{\prime}(u)=0$ and $B_{i}^{\prime}(u)=0$. From $B_{i}^{\prime}(u)=0$, we have $a_{i} T_{0}^{\prime}\left(t_{i}, \alpha_{i}\right)=0\left(0<t_{i}<\pi / 2\right)$,i,e. $T_{0}^{\prime}\left(t_{i}, \alpha_{i}\right)=0$. Obviously, this can lead to contradictions. Thus, we can get $Q_{i}^{\prime}(u) \neq 0$, and we also can easily get the curve segments $Q_{i}(u)$ have not cusps and inflection points.

Next, we consider the inflection points. Let $H(t)=\operatorname{det}\left(Q_{i}^{\prime}(u), Q_{i}^{\prime \prime}(u), Q_{i}^{\prime \prime}(u)\right)$, we have

$$
\begin{aligned}
& H(t)=\operatorname{det}\left[\sum_{j=i-3}^{i} B_{j}^{\prime}(u) P_{j} \quad \sum_{j=i-3}^{i} B_{j}^{\prime \prime}(u) P_{j} \quad \sum_{j=i-3}^{i} B_{j}^{\prime \prime \prime}(u) P_{j}\right] \\
& =\left|\begin{array}{cccc}
\sum_{j=i-3}^{i} B_{j}(u) & \sum_{j=i-3}^{i} B_{j}^{\prime}(u) & \sum_{j=i-3}^{i} B_{j}^{\prime \prime}(u) & \sum_{j=i-3}^{i} B_{j}^{\prime \prime \prime}(u) \\
\sum_{j=i-3}^{i} B_{j}(u) P_{j} & \sum_{j=i-3}^{i} B_{j}^{\prime}(u) P_{j} & \sum_{j=i-3}^{i} B_{j}^{\prime \prime}(u) P_{j} & \sum_{j=i-3}^{i} B_{j}^{\prime \prime \prime}(u) P_{j}
\end{array}\right| \\
& =\left|\left[\begin{array}{cccc}
1 & 1 & 1 & 1 \\
P_{i-3} & P_{i-2} & P_{i-1} & P_{i}
\end{array}\right]\left[\begin{array}{cccc}
B_{i-3}(u) & B_{i-3}^{\prime}(u) & B_{i-3}^{\prime \prime}(u) & B_{i-3}^{\prime \prime \prime}(u) \\
B_{i-2}(u) & B_{i-2}^{\prime}(u) & B_{i-2}^{\prime \prime}(u) & B_{i-2}^{\prime \prime \prime}(u) \\
B_{i-1}(u) & B_{i-1}^{\prime}(u) & B_{i-1}^{\prime \prime}(u) & B_{i-1}^{\prime \prime \prime}(u) \\
B_{i}(u) & B_{i}^{\prime}(u) & B_{i}^{\prime \prime}(u) & B_{i}^{\prime \prime}(u)
\end{array}\right]\right| \\
& =\left\lfloor\left[\begin{array}{cccc}
1 & 0 & 0 & 0 \\
P_{i-3} & b_{i-2} & b_{i-1} & b_{i}
\end{array}\right]\left[\begin{array}{cccc}
B_{i-3}(u) & B_{i-3}^{\prime}(u) & B_{i-3}^{\prime \prime}(u) & B_{i-3}^{\prime \prime \prime}(u) \\
B_{i-2}(u) & B_{i-2}^{\prime}(u) & B_{i-2}^{\prime \prime}(u) & B_{i-2}^{\prime \prime \prime}(u) \\
B_{i-1}(u) & B_{i-1}^{\prime}(u) & B_{i-1}^{\prime \prime}(u) & B_{i-1}^{\prime \prime \prime}(u) \\
B_{i}(u) & B_{i}^{\prime}(u) & B_{i}^{\prime \prime}(u) & B_{i}^{\prime \prime \prime}(u)
\end{array}\right] \mid\right. \\
& =\left(b_{i-2}, b_{i-1}, b_{i}\right) M_{i}(u) \text {. }
\end{aligned}
$$

Where, $\left(b_{i-2}, b_{i-1}, b_{i}\right)$ is mixed product of vector edge $b_{i-2}, b_{i-1}, b_{i}$, by directly computing, we have $M_{i}(t)>0$. For any $u \in\left[u_{i}, u_{i+1}\right]$, since $\left(b_{i-2}, b_{i-1}, b_{i}\right) \neq 0$, we can get $H(t) \neq 0$, and it has same positive and negative property as $\left(b_{i-2}, b_{i-1}, b_{i}\right)$. Thus, $Q_{i}(u)$ has not inflection points, and it has the same rotation direction as the control points.

6.3.2 Shape analysis of the planar QCT-B spline curve

If the four control points $\left(P_{j} \in R^{2}, j=i-3, i-2, i-1, i\right)$ are coplanar, $Q_{i}(u)$ is planar curve, at this time, we have the following theorem about its shape features.

Theorem 14 When $b_{i} \| b_{i-2}$, the planar QCT-B spline curve $Q_{i}(u)$ has not cusps and loops; If and only if 
$b_{i}$ has the same direction of $b_{i-2}, Q_{i}(u)$ only has one inflection point; When $b_{i} \not \mid b_{i-2}$, let $b_{i-1}=U b_{i-2}+V b_{i}$, where $(U, V)=\left(b_{i-1} \times b_{i}, b_{i-2} \times b_{i-1}\right) /\left(b_{i-2} \times b_{i}\right)$, then the shape feature of $Q_{i}(u)$ depends on the following distribution of points $(U, V)$ in the $U V$-plane (see Table 2), ie

Table 2 Distribution of shape features of planar QCT-B spline curves

\begin{tabular}{ccccc}
\hline \multirow{2}{*}{$(U, V)$} & \multicolumn{4}{c}{ The shape features of planar QCT-B spline curve segment } \\
\cline { 2 - 5 } & Convexity & Cusps & Loops & Inflection points \\
\hline$C$ & & 1 & No & No \\
$L$ & & No & 1 & No \\
$S$ & No & No & 1 \\
$D$ & Global convex & No & No & 2 \\
$N_{0}$ & Locally convex & No & No & No \\
$N_{1} \cup N_{2}$ & & & & No \\
\hline
\end{tabular}

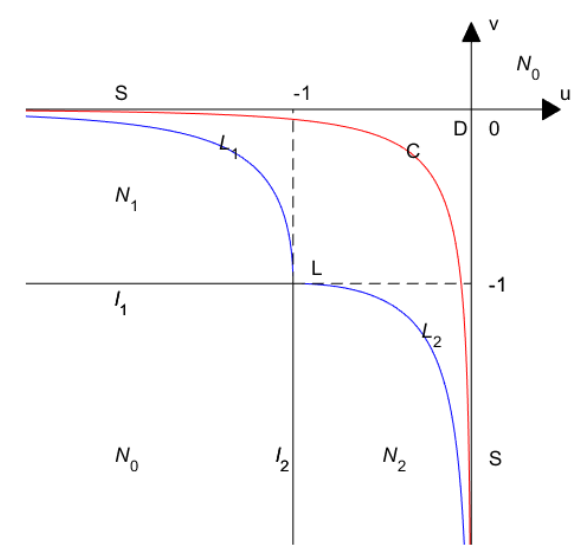

Fig.10 The complete shape analysis diagram of the QCT-B spline curve

Where, the description of each distribution area is as follows: $S=\{(U, V) \mid U V<0\} \cup\{(U .0) \mid-1<U<0\}$ $\cup\{(0, V) \mid-1<V<0\} ; D$ is an open region surrounded by coordinate the axis $U, V$ and curve $C$. $L$ is an region surrounded the curve $L_{1}, L_{2}$ and $C$, where $L_{1} \subset L, L_{2} \subset L$, but $C \not \subset L ; N_{1}$ is an open region surrounded the curve $L_{1}$ and $l_{1} ; N_{2}$ is an open region surrounded the curve $L_{2}$ and $l_{2}$; $N_{0}=R^{2} \backslash\left(S \cup D \cup C \cup L \cup N_{1} \cup N_{2}\right) \quad$, which include the boundaries $\{(U, 0) \mid U(U+1) \geq 0\} \cup$ $\{(0, V) \mid V(V+1) \geq 0\} \cup l_{1} \cup l_{2}$. The parametric equations for the relevant curves are as follows:

$$
\begin{aligned}
& C:\left\{\begin{array}{l}
U=\frac{d_{i} T_{3}^{\prime}\left(t_{i} ; \beta_{i}\right)}{\left(a_{i}+b_{i 0}-b_{i 1}\right) T_{0}^{\prime}\left(t_{i} ; \alpha_{i}\right)+\left(b_{i 3}-b_{i 2}\right) T_{3}^{\prime}\left(t_{i} ; \beta_{i}\right)+2\left(b_{i 2}-b_{i 1}\right) \sin t \cos t} \quad 0<t_{i}<\pi / 2 \\
V=-\frac{a_{i} T_{0}^{\prime}\left(t_{i} ; \alpha_{i}\right)}{\left(a_{i}+b_{i 0}-b_{i 1}\right) T_{0}^{\prime}\left(t_{i} ; \alpha_{i}\right)+\left(b_{i 3}-b_{i 2}\right) T_{3}^{\prime}\left(t_{i} ; \beta_{i}\right)+2\left(b_{i 2}-b_{i 1}\right) \sin t \cos t}
\end{array}\right. \\
& L_{1}:\left\{\begin{array}{l}
U=\frac{d_{i} T_{3}\left(t_{i} ; \beta_{i}\right)}{\left(a_{i}+b_{i 0}\right) T_{0}\left(t_{i} ; \alpha_{i}\right)+b_{i 1} T_{1}\left(t_{i} ; \alpha_{i}\right)+b_{i 2} T_{2}\left(t_{i} ; \beta_{i}\right)+b_{i 3} T_{3}\left(t_{i} ; \beta_{i}\right)-b_{i 0}-a_{i}} 0<t_{i}<\pi / 2 \\
V=\frac{a_{i}-a_{i} T_{0}\left(t_{i} ; \alpha_{i}\right)}{\left(a_{i}+b_{i 0}\right) T_{0}\left(t_{i} ; \alpha_{i}\right)+b_{i 1} T_{1}\left(t_{i} ; \alpha_{i}\right)+b_{i 2} T_{2}\left(t_{i} ; \beta_{i}\right)+b_{i 3} T_{3}\left(t_{i} ; \beta_{i}\right)-b_{i 0}-a_{i}}
\end{array}\right. \\
& L_{2}:\left\{\begin{array}{l}
U=\frac{d_{i}-d_{i} T_{3}\left(t_{i} ; \beta_{i}\right)}{b_{i 3}-\left[\left(a_{i}+b_{i 0}\right) T_{0}\left(t_{i} ; \alpha_{i}\right)+b_{i 1} T_{1}\left(t_{i} ; \alpha_{i}\right)+b_{i 2} T_{2}\left(t_{i} ; \beta_{i}\right)+b_{i 3} T_{3}\left(t_{i} ; \beta_{i}\right)\right]} 0<t_{i}<\pi / 2 \\
V=\frac{a_{i} T_{0}\left(t_{i} ; \alpha_{i}\right)}{b_{i 3}-\left[\left(a_{i}+b_{i 0}\right) T_{0}\left(t_{i} ; \alpha_{i}\right)+b_{i 1} T_{1}\left(t_{i} ; \alpha_{i}\right)+b_{i 2} T_{2}\left(t_{i} ; \beta_{i}\right)+b_{i 3} T_{3}\left(t_{i} ; \beta_{i}\right)\right]}
\end{array}\right. \\
& l_{1}: V=\frac{V^{*}(U+1)}{U^{*}+1},-1<U<U^{*}
\end{aligned}
$$




$$
l_{2}: V=-1+\frac{U\left(V^{*}+1\right)}{U^{*}}, U^{*}<U<0
$$

Where $U^{*}=V^{*}=\frac{d_{i}}{b_{i 3}-b_{i 0}-a_{i}}$.

Proof We first consider the situation $b_{i} \| b_{i-2}$, we can easily get $b_{i-1}=U b_{i-2}+V b_{i}$, then we combine (29), we have

$$
Q_{i}(u)=P_{i-3}+\left\{1-B_{i-3}(u)+U\left[B_{i-1}(u)+B_{i}(u)\right]\right\} b_{i-2}+\left\{B_{i}(u)+V\left[B_{i-1}(u)+B_{i}(u)\right]\right\} b_{i} .
$$

Below we discuss the cusps, inflection points, loops and convexity.

1) Cusps

The necessary condition that the planar QCT-B spline curve $Q_{i}(u)$ has cusps is $Q_{i}^{\prime}(u)=0$ $\left(u_{i}<u<u_{i+1}\right)$. From (35), we have

$$
\left\{U\left[B_{i-1}^{\prime}(u)+B_{i}^{\prime}(u)\right]-B_{i-3}^{\prime}(u)\right\} b_{i-2}+\left\{B_{i}^{\prime}(u)+V\left[B_{i-1}^{\prime}(u)+B_{i}^{\prime}(u)\right]\right\} b_{i}=0
$$

Since $b_{i-2}$ and $b_{i}$ are linear independent, according to (36), we can get the curve $C$ :

$$
C:\left\{\begin{array}{l}
U=\frac{B_{i-3}^{\prime}(u)}{B_{i-1}^{\prime}(u)+B_{i}^{\prime}(u)} \\
V=-\frac{B_{i}^{\prime}(u)}{B_{i-1}^{\prime}(u)+B_{i}^{\prime}(u)}
\end{array} u_{i}<u<u_{i+1}\right.
$$

We analyze the shape of the curve $C$ given in (30) and (37), we have

$$
\begin{aligned}
& \lim _{u \rightarrow u_{i}} U=-1, \lim _{v \rightarrow u_{i}} V=0, \\
& \lim _{u \rightarrow u_{i}} U=0, \lim _{v \rightarrow u_{i+1}} V=-1 .
\end{aligned}
$$

This shows $C$ has two asymptotes $U=0$ and $V=0$. By direct calculation, we have

$$
\frac{d U}{d V}<0, \frac{d^{2} U}{d V^{2}}>0
$$

This indicates that $C$ is a monotonically decreasing and strictly convex curve, and the curve is tangent to the axis $U$ at $(-1,0)$, and it is tangent to the axis $V$ axis at $(0,-1)$.

For any $\left(U_{0}, V_{0}\right) \in C$, we have $Q_{i}^{\prime}(u)=0$ and $Q_{i}^{\prime \prime}(t) \neq 0$. In fact, similar to the discussion of (36) and (37), we have

$$
\left\{U\left[B_{i-1}^{\prime \prime}(u)+B_{i}^{\prime \prime}(u)\right]-B_{i-3}^{\prime \prime}(u)\right\} b_{i-2}+\left\{B_{i}^{\prime \prime}(u)+V\left[B_{i-1}^{\prime \prime}(u)+B_{i}^{\prime \prime}(u)\right]\right\} b_{i}=0
$$

In fact, similar to the discussion of 4.4.2, no parameters $u \in\left[u_{i}, u_{i+1}\right]$ can be satisfied (36) and (38) at the same time, which indicates $Q_{i}^{\prime}(u)=0$ and $Q_{i}^{\prime \prime}(u) \neq 0$. Thus, we have

$$
Q_{i}^{\prime}(u)=Q_{i}^{\prime \prime}(u)\left(u-u_{0}\right)+o\left(u-u_{0}\right) .
$$

We can know that $Q_{i}^{\prime}(u)$ change the sign at $u_{0}$, thus, $Q_{i}\left(u_{0}\right)$ is a cusp. Therefore, the planar QCT-B spline curve defined by (26) possess cusps are equivalent to $(u, v) \in C$.

2) Inflection points

$$
Q_{i}\left(u_{0}\right) \text { is a inflection point of the QCT-B spline curve means that } Q_{i}^{\prime}(u) \times Q_{i}^{\prime \prime}(u)=
$$
$g(u ; U, V)\left(b_{i-3} \times b_{i}\right)$ change its sign when the curve passes the point $u_{0}$. Where,

$$
g(u ; U, V)=-\left|\begin{array}{ll}
Q_{i-3}^{\prime}(u) & Q_{i}^{\prime}(u) \\
Q_{i-3}^{\prime \prime}(u) & Q_{i}^{\prime \prime}(u)
\end{array}\right|+U\left|\begin{array}{ll}
Q_{i-1}^{\prime}(u) & Q_{i}^{\prime}(u) \\
Q_{i-1}^{\prime \prime}(u) & Q_{i}^{\prime \prime}(u)
\end{array}\right|+V\left|\begin{array}{ll}
Q_{i-3}^{\prime}(u) & Q_{i-2}^{\prime}(u) \\
Q_{i-3}^{\prime \prime}(u) & Q_{i-2}^{\prime \prime}(u)
\end{array}\right| .
$$

Since $b_{i-3} \times b_{i} \neq 0$, we only consider the sign of $g(u ; U, V)$. On the $U V-$ planar, the possible region that makes the curve $Q_{i}(u)$ have inflection points must be covered by the line family $g(u ; U, V)$. From the previous discussion, it can be seen that the envelope of the line family $g(u ; U, V)$ is curve $C$, and the curve $C$ is globally convex, so the region swept by the tangent of the curve is $S \cup D \cup C$, which also is the possible region of the inflection points, where $S=\{(U, V) \mid U V<0\} \cup\{(U, 0) \mid-1<U<0\} \cup\{(0, V) \mid-1<V<0\}, \quad D$ is the open 
region enclosed by the curve $C$ and the coordinate axis $\mathrm{U}, \mathrm{V}$ (see Fig.10). At any point $\left(U_{0}, V_{0}\right) \in S \cup D \cup C$ has at least one straight line on the $U V$-plane that is tangent to the curve $C$. When $\left(U_{0}, V_{0}\right) \in C$, according to Taylor expansion, we have $g(u ; U, V)=0.5 g_{u u}^{\prime \prime}\left(u_{0} ; U_{0}, V_{0}\right)\left(u-u_{0}\right)^{2}+o\left(u-u_{0}\right)^{2}$, where $g_{u u}^{\prime \prime}\left(u_{0} ; U_{0}, V_{0}\right) \neq 0$. We can get that $g(u ; U, V)$ will not change the sign when the curve pass the parameter $u_{0}$. Thus, $Q_{i}(u)$ has not inflection points; When $\left(U_{0}, V_{0}\right) \in S \cup D$, let $g\left(u_{0} ; U, V\right)$ is one of a tangent line of the curve $C$. Thus, we have $g\left(u ; U_{0}, V_{0}\right)=g_{u}^{\prime}\left(u_{0} ; U_{0}, V_{0}\right)\left(u-u_{0}\right)+o\left(u-u_{0}\right)$ (where $g_{u}^{\prime}\left(u_{0} ; U_{0}, V_{0}\right) \neq 0$, otherwise $\left.\left(U_{0}, V_{0}\right) \in C\right)$, we can get that $g\left(u ; U_{0}, V_{0}\right)$ change sign at $u_{0}$; Further, when $\left(U_{0}, V_{0}\right) \in S$, only one tangent of curve $C$ can be made through it, and $Q_{i}(u)$ has only one inflection point; When $\left(U_{0}, V_{0}\right) \in D$, there are two tangent of curve $C$ can be made, and $Q_{i}(u)$ has two inflection point.

3) Loops

The non-uniform QCT-B spline curve has loops means that $Q_{i}\left(u_{1}^{*}\right)=Q_{i}\left(u_{2}^{*}\right)$ when $u_{i} \leq u_{1}^{*}<u_{2}^{*} \leq u_{i+1}$. This is equivalent to $U, V, u_{1}^{*}, u_{2}^{*}$ satisfying the following equations:

$$
\left\{\begin{array}{l}
U=\frac{B_{i-3}\left(u_{2}^{*}\right)-B_{i-3}\left(u_{1}^{*}\right)}{B_{i-1}\left(u_{2}^{*}\right)+B_{i}\left(u_{2}^{*}\right)-B_{i-1}\left(u_{1}^{*}\right)-B_{i}\left(u_{1}^{*}\right)},\left(u_{1}^{*}, u_{2}^{*}\right) \in \Delta \\
V=\frac{B_{i}\left(u_{1}^{*}\right)-B_{i}\left(u_{2}^{*}\right)}{B_{i-1}\left(u_{2}^{*}\right)+B_{i}\left(u_{2}^{*}\right)-B_{i-1}\left(u_{1}^{*}\right)-B_{i}\left(u_{1}^{*}\right)}
\end{array}\right.
$$

Where $\Delta=\left\{\left(u_{1}^{*}, u_{2}^{*}\right) \in R^{2} \mid u_{i} \leq u_{1}^{*}<u_{2}^{*} \leq u_{i+1}\right\}$. The equations given in (40) define a topological mapping $G: \Delta \subset R^{2} \rightarrow G(\Delta) \subset R^{2}$. Thus, the image $L=F(\Omega)$ is a simply connected region in $U V-$ plane. The three boundary lines of $L$ correspond to the three boundary lines $u_{1}^{*}=u_{2}^{*}, u_{1}^{*}=u_{i}$ and $u_{2}^{*}=u_{i+1}$ of $\Delta$, i.e. the curve $C$ (not belonging to $L$ ), $L_{1}$ and $L_{2}$ (both belonging to $L$ ). The curve $Q_{i}(u)$ corresponding to point $(U, V)$ in $L$ only has one loop. From the mathematical analysis, it can be inferred that both the curves $L_{1}$ and $L_{2}$ are continuous functions of monotonically decreasing and strictly convex, and when $u \rightarrow u_{i}$, the $U$ axis is tangent of the curve $L_{1}$ at the point $(-1,0)$, and when $u \rightarrow u_{i+1}$, the $V$ axis is tangent of the curve $L_{2}$ at the point $(0,-1) . L_{1}$ and $L_{2}$ intersect at point $\left(U^{*}, V^{*}\right)$, the tangent line $l_{2}$ of $L_{1}$ at point $\left(U^{*}, V^{*}\right)$ crosses point $(0,-1)$, and the tangent line $l_{1}$ of $L_{2}$ at point $\left(U^{*}, V^{*}\right)$ crosses point $(-1,0)$ (see Fig.10), where the equation of $l_{1}$ and $l_{2}$ given in (33) and (34), respectively.

4) Convexity

The following is the case for $(U, V) \in N=R^{2} \backslash(C \cup S \cup D \cup L)$. Obviously, the curve $Q_{i}(u)$ has not cusps, inflection points and loops. Next, we consider $R(u)=Q^{\prime}\left(u_{i}\right) \times\left[Q(u)-Q\left(u_{i}\right)\right]$ and $S(u)=\left[Q(u)-Q\left(u_{i}\right)\right] \times Q^{\prime}(u)$, from (35), we have

$$
\left\{\begin{array}{l}
R(u)=\Psi(u ; U, V)\left(b_{i-3} \times b_{i}\right) \\
S(u)=\Phi(u ; U, V)\left(b_{i-3} \times b_{i}\right)
\end{array}\right.
$$

where,

$$
\begin{aligned}
& \Psi(u ; U, V)=\left(\alpha_{i}+3\right)\left\{U\left[\left(b_{i 1}-b_{i 0}\right) B_{i}+a_{i}\left(B_{i-1}-b_{i 1}\right)\right]+B_{i-3}\left[a_{i}-V\left(c_{i 1}-c_{i 0}\right)\right]\right\} \\
& \Phi(u ; U, V)=\left[B_{i-1}\left(u_{i}\right)-B_{i-3}(u)\right] B_{i}^{\prime}(u)+B_{i-3}^{\prime}(u) B_{i}(u) \\
& +U\left\{\left[B_{i-1}(u)+B_{i}(u)-B_{i-1}\left(u_{i}\right)\right] B_{i}^{\prime}(u)-\left[B_{i-1}^{\prime}(u)+B_{i}^{\prime}(u)\right] B_{i}(u)\right\} \\
& +V\left\{\left[B_{i-1}\left(u_{i}\right)-B_{i-3}(u)\right]\left[B_{i-1}^{\prime}(u)+B_{i}^{\prime}(u)\right]+B_{i-3}^{\prime}(u)\left[B_{i-1}(u)+B_{i}(u)-B_{i-1}\left(u_{i}\right)\right]\right\}
\end{aligned}
$$

According to (41), $\Psi(u ; U, V)=0$ determines a family of straight lines passing $(-1,0)$ on the $U V$-plane. Its slope is $K(u)=\frac{\left(b_{i 1}-b_{i 0}\right) B_{i}(u)+a_{i}\left(B_{i-1}(u)-b_{i 1}\right)}{B_{i-3}(u)}$, by direct computing,we have $\frac{V\left(c_{i 1}-c_{i 0}\right)-a_{i}}{U}<$ $K(u)<0$, therefore, the region swept by the line family $\Psi(u ; U, V)=0$ in $N$ happens to be the part enclosed by the curve $L_{1}$ and the straight line segment $l_{1}$, which we record as $N_{1}$ (excluding the boundary lines $L_{1}$ and $l_{1}$, as shown in Fig. 10). If $\left(U_{0}, V_{0}\right) \in N_{1}$, we have $\Psi^{\prime}(u ; U, V) \neq 0$ (otherwise $\left.U=-1, V=0\right)$. Thus, from the expansion $\Psi\left(u ; U_{0}, V_{0}\right)=\Psi^{\prime}\left(u_{0} ; U_{0}, V_{0}\right)\left(u-u_{0}\right)+o\left(u-u_{0}\right)$, we can know that $\Psi\left(u ; U_{0}, V_{0}\right)$ will change sign 
at point $u_{0}$. Actually, the region $N_{1}$ is exactly the portion of the tangent of the curve $L_{2}$ swept in $N$.

Solve the following equations about $U V$ :

$$
\left\{\begin{array}{l}
\Phi(u ; U, V)=0, \\
\Phi^{\prime}(u ; U, V)=0 .
\end{array}\right.
$$

We can easily check that the solution is exactly is the equation of the parameters curve $L_{1}$. The region where the tangent of $L_{1}$ is swept in $N$ is $N_{2}$. Then $N_{2}$ is surrounded by the curve $L_{2}$ and the straight line segment $l_{2}$ (where $L_{2} \not \subset N_{2}, l_{2} \not \subset N_{2}$, see Fig. 10). If $\left(U_{0}, V_{0}\right) \in N_{2}$, we have $\Phi^{\prime}\left(u_{0} ; U_{0}, V_{0}\right) \neq 0$ (otherwise $\left.\left(U_{0}, V_{0}\right) \in L_{1}\right)$. Thus, from $\Phi\left(u ; U_{0}, V_{0}\right)=\Phi^{\prime}\left(u_{0} ; U_{0}, V_{0}\right)\left(u-u_{0}\right)+o\left(u-u_{0}\right)$, we can easily check that $\Phi\left(u ; U_{0}, V_{0}\right)$ will change the sign at the point $u_{0}$.

Let $N_{0}=N \backslash\left(N_{1} \cup N_{2}\right)$, when $(U, V) \in N_{0}, Q_{i}^{\prime}(u) \times Q_{i}^{\prime \prime}(u), R(u)$ and $S(u)$ all doesn't change sign. Thus, the curve $Q_{i}(u)$ is global convex; When $(U, V) \in N_{1}, Q_{i}^{\prime}(u) \times Q_{i}^{\prime \prime}(u)$ and $S(u)$ doesn't change sign, but $R(u)$ change sign one time, at this time, the curve $Q_{i}(u)$ is locally convex; When $(U, V) \in N_{2}, Q_{i}^{\prime}(u) \times Q_{i}^{\prime \prime}(u)$ and $R(u)$ doesn't change sign, but $S(u)$ change sign one time, at this time, the curve $Q_{i}(u)$ is locally convex[34].

Finally, when $b_{i-2} \| b_{i}$, the curve $Q_{i}(u)$ has not cusps and loops; If and only if when $b_{i-2}$ has the same direction of $b_{i}, Q_{i}(u)$ only has one inflection point.

5) Adjustment effect of shape parameters

According to the equation (30) (34) and region division diagram of the non-uniform QCT B spline curve, the following conclusions can be drawn:

(1) The change of shape parameters $\alpha_{i}$ and $\beta_{i}$ does not affect the single inflection point region $S$ and global convex region $N$, so when there is only one inflection point on $Q_{i}(u)$, shape parameters cannot be adjusted to eliminate it.

(2) The change of shape parameters $\alpha_{i}$ and $\beta_{i}$ does not affect the region $N_{0} / Z$, in this time, $Q_{i}(u)$ is global convex, where $Z$ is the triangular region enclosed by $(-1,0),(0,-1),\left(U^{*}, V^{*}\right)$ (including the boundary line $l_{1}$ and $l_{2}$, excluding the straight line connecting two points $(-1,0)$ and $\left.(0,-1)\right)$.

(3) With the increase of shape parameters $\alpha_{i}, \beta_{i} \in(-1,1],\left(U^{*}, V^{*}\right)$ tends to $(-1 / 14,-1 / 14)$, and with the decrease of shape parameters, $\left(U^{*}, V^{*}\right)$ tends to $(-1 / 2,-1 / 2)$. The region of double inflection points $D$ and loops $L$ gradually shrinks, and the global convex region $Z$ gradually expands, see[11].
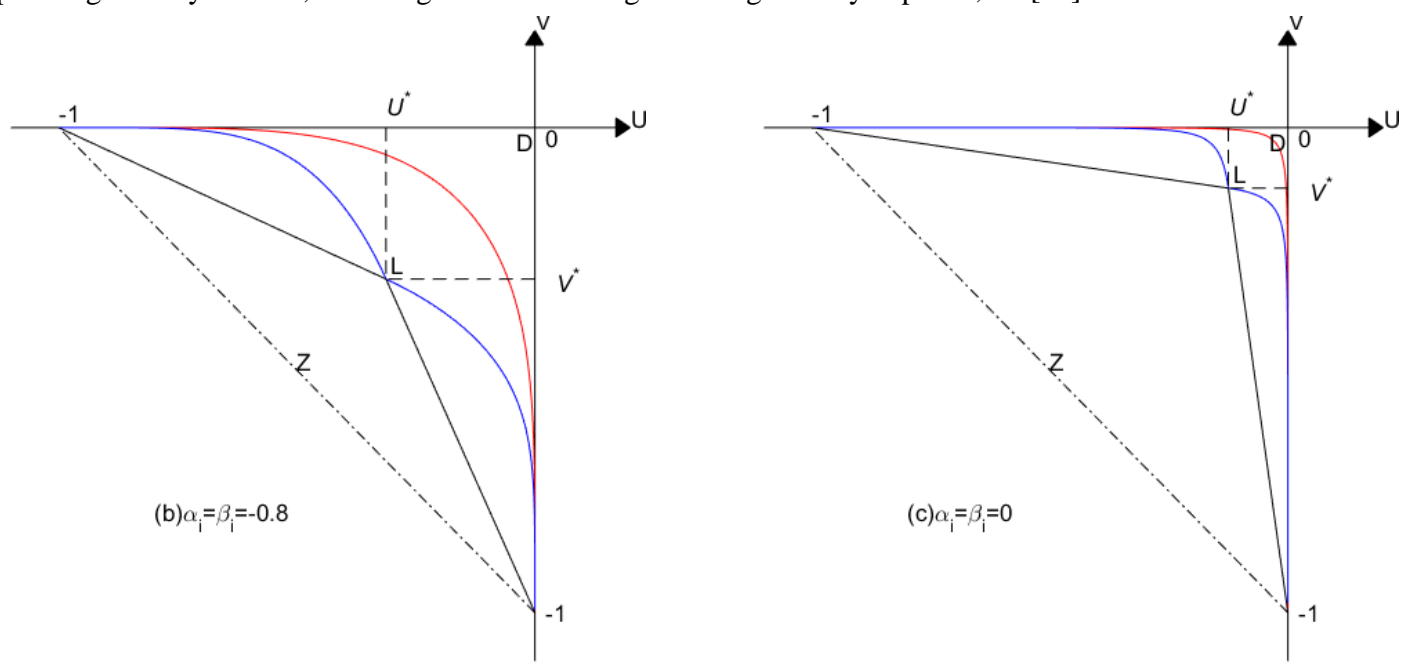


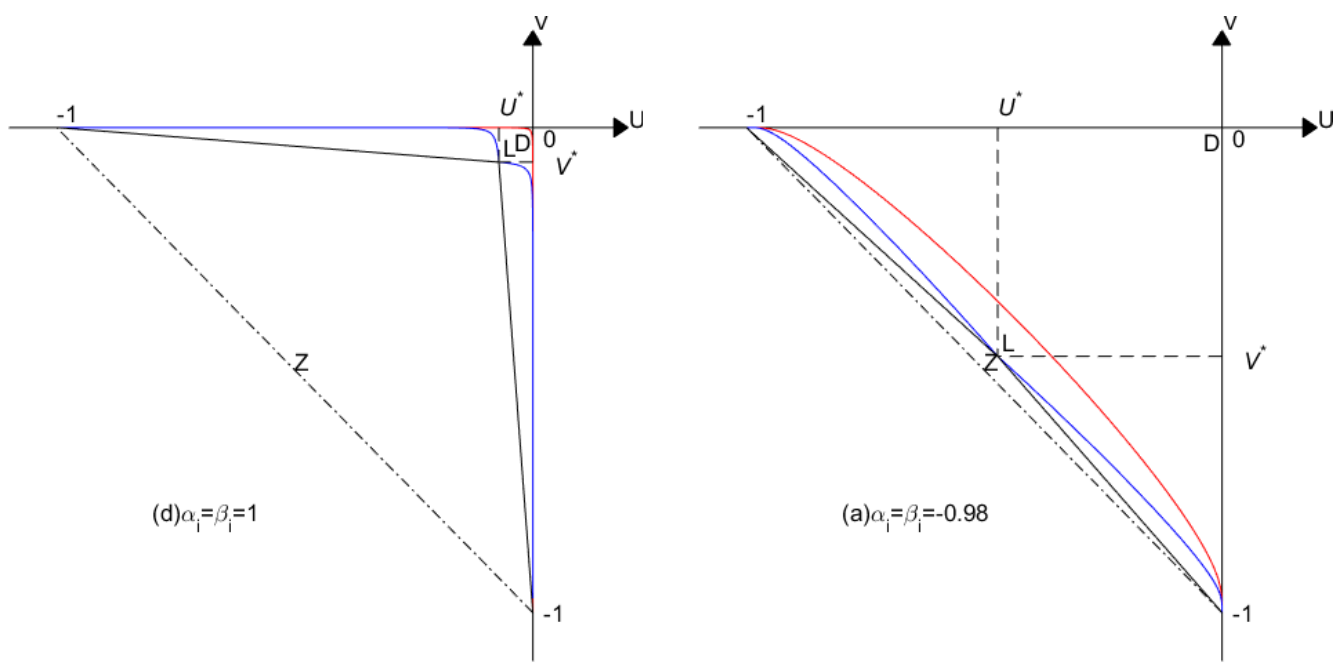

Fig.11 The effect of parameters $\alpha_{i}$ and $\beta_{i}$ on $L, D, C, Z$

(4) For any $\alpha_{i}, \beta_{i} \in(-1,1]$, the curve all exists locally convex region $N_{1} \cup N_{2}$, but when $\alpha_{i}, \beta_{i} \rightarrow-1$ at the same time, $N_{1} \cup N_{2} \rightarrow 0$, see Fig.12.
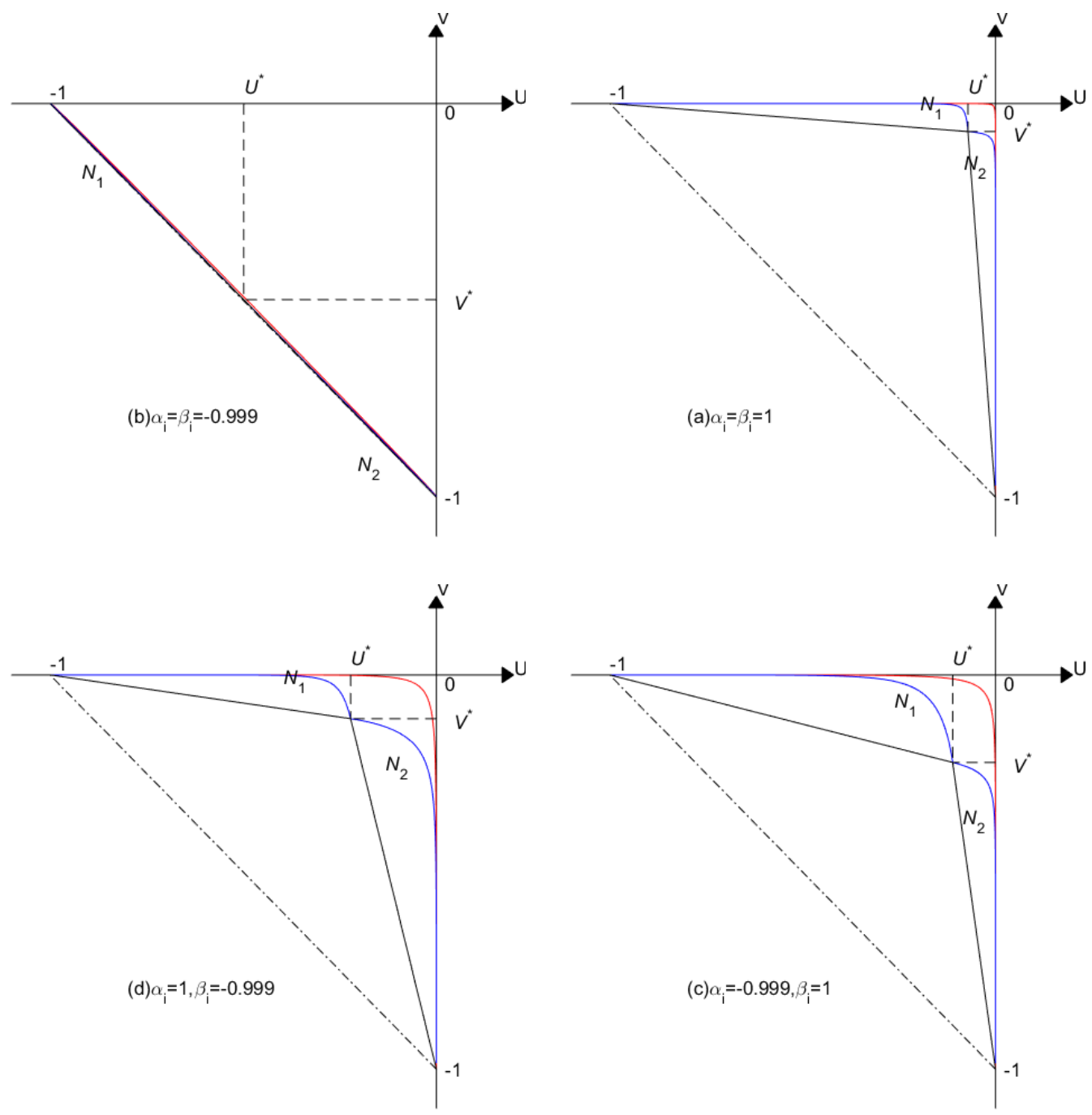

Fig.12 The influence of $\alpha_{i}$ and $\beta_{i}$ on $N_{1} \cup N_{2}$

(5) By increasing the value of the shape parameter $\alpha_{i}, \beta_{i}$, the cusp, loop and double inflection point on the 
curve can be eliminated, and the curve can be adjusted to global convexity, see Fig.13.
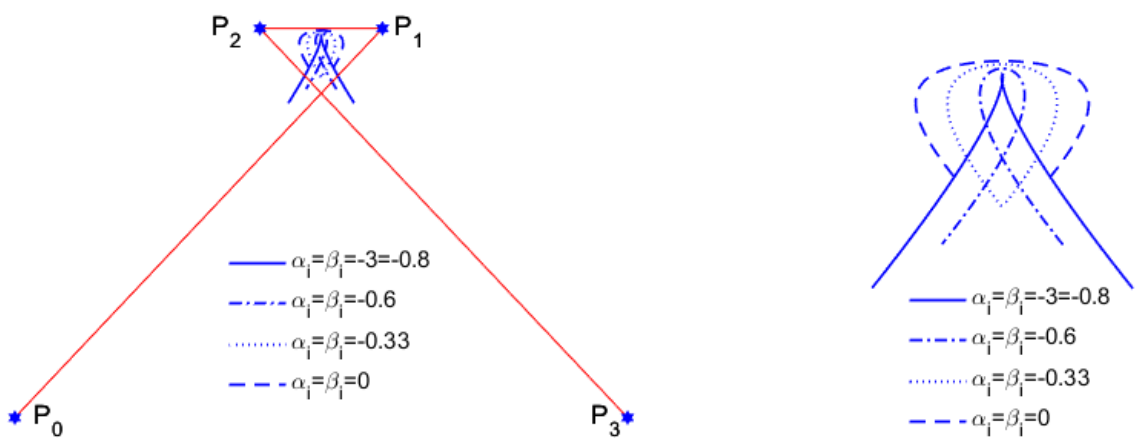

Fig. 13 The unwanted shape features is avoided

by adjusting shape parameters(Left: the original image, Right: enlarge image)

\section{Non-uniform QCT-B spline surface}

Definition 7 Given control points $P_{i j}(i=0,1, \ldots, m, j=0,1, \ldots, n) \in R^{3}$ and two knot vector $U=\left(u_{0}, u_{1}, \ldots\right.$, $\left.u_{n+4}\right), V=\left(v_{0}, v_{1}, \ldots, v_{n+4}\right)$, for any $\alpha_{i j}, \beta_{i j} \in[-1,1], m, n \geq 3, u \in\left[u_{3}, u_{m+1}\right], v \in\left[v_{3}, v_{n+3}\right]$, we call

$$
B(u, v)=\sum_{i=0}^{m} \sum_{j=0}^{n} B_{i}(u) B_{j}(v) P_{i j}
$$

as a non-uniform QCT-B spline surface with four shape parameters. Where, $B_{i}(u)$ and $B_{j}(v)$ are non-uniform B spline basis with shape parameters $\alpha_{i 1}, \beta_{i 1}$ and $\alpha_{j 2}, \beta_{j 2}$, respectively.

The surface slice defined in $\left[u_{i}, u_{i+1}\right] \times\left[v_{j}, v_{j+1}\right]$ is

$$
B_{i j}(u, v)=\sum_{s=i-3}^{i} \sum_{t=j-3}^{j} B_{s}(u) B_{t}(v) P_{s t} .
$$

In fact, in addition to variation diminishing, the properties of QCT-B spline curves can be extended to QCT-B spline surface. Due to space limitation, we will not discuss it in detail here, but the higher-order continuity of uniform QCT-B spline surface is given below.

Theorem 15 Given two uniform knot vector $U$ and $V$, when shape parameters satisfy $\alpha_{i 1}, \beta_{i 1}, \alpha_{j 2}, \beta_{j 2} \in$ $[-1,1]$ and $\alpha_{i+1,1}=\beta_{i 1}, \alpha_{j+1,2}=\beta_{j 2}$, the uniform QCT-B spline surface are $C^{2 n-1}(n=1,2,3, \cdots)$ continuity at each knot.

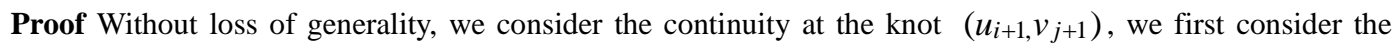
continuity of the $u$ direction, and the continuity of the $v$ direction can be discussed similarly. For $u \in\left[u_{i}, u_{i+1}\right), v \in\left[v_{j}, v_{j+1}\right), i=3,4, \ldots, m, j=3,4, \ldots, n$, we have

$$
\begin{array}{ll}
B_{i}(u)=a_{i} A_{0}\left(t_{i} ; \alpha_{i}\right), & B_{i-1}(u)=\sum_{k=0}^{3} b_{i, k} A_{k}\left(t_{i} ; \alpha_{i}, \beta_{i}\right), \\
B_{i-2}(u)=\sum_{k=0}^{3} c_{i, k} A_{k}\left(t_{i} ; \alpha_{i}, \beta_{i}\right), & B_{i-3}(u)=d_{i} A_{3}\left(t_{i}, \beta_{i}\right), \\
B_{j}(u)=a_{j} A_{0}\left(t_{j} ; \alpha_{j}\right), & B_{j-1}(u)=\sum_{l=0}^{3} b_{j, l} A_{l}\left(t_{j} ; \alpha_{j}, \beta_{j}\right), \\
B_{j-2}(u)=\sum_{l=0}^{3} c_{j, l} A_{l}\left(t_{j} ; \alpha_{j}, \beta_{j}\right), & B_{j-3}(u)=d_{j} A_{3}\left(t_{j}, \beta_{j}\right) .
\end{array}
$$

Therefore,

$$
\begin{gathered}
\frac{\delta^{2 n-1} B_{i j}\left(u_{i+1}^{-}, v_{j+1}\right)}{\delta u^{2 n-1}}=\sum_{s=i-3}^{i} \sum_{t=j-3}^{j}\left[\frac{\delta^{2 n-1} B_{s}\left(u_{i+1}^{-}\right)}{\delta u^{2 n-1}}\right] B_{t}\left(v_{j+1}\right) P_{s t}, \\
\frac{\delta^{2 n-1} B_{i j}\left(u_{i+1}^{+}, v_{j+1}\right)}{\delta u^{2 n-1}}=\sum_{s=i-3}^{i} \sum_{t=j-3}^{j}\left[\frac{\delta^{2 n-1} B_{s}\left(u_{i+1}^{+}\right)}{\delta u^{2 n-1}}\right] B_{t}\left(v_{j+1}\right) P_{s t},
\end{gathered}
$$


From the Theorem 8 and $\alpha_{i+1,1}=\beta_{i 1}, \alpha_{j+1,2}=\beta_{j 2}$, we have

$$
\frac{\delta^{2 n-1} B_{i j}\left(u_{i+1}^{-}, v_{j+1}\right)}{\delta u^{2 n-1}}=\frac{\delta^{2 n-1} B_{i j}\left(u_{i+1}^{+}, v_{j+1}\right)}{\delta u^{2 n-1}} .
$$

Theorem is proved.

\section{Conclusion}

When the traditional literature improves the Bézier method and the B-spline method, it only focuses on whether the curve flexibility can be increased. Therefore, the constructed curves and surfaces retain only some basic properties of the Bézier method and the B-spline method, such as convex hull and affine invariance, symmetry, etc., such as totally positive property, variation diminishing property and shape preservation are overlooked. In view of the problems of traditional improved methods, this paper constructs a set of optimal norm-positive basis from the property of shape preservation, and designs curve and surfaces with high-order continuity. A lot of discussion and analysis show that the curve and surface constructed in this paper not only retains the good properties of the traditional Bézier method and the B-spline method, but also has shape preservation, shape adjustability and high-order continuity, which is suitable for curve and surface design. In addition, the shape of the curve, such as sharp points, inflection points, loops, convexity, etc., is analyzed in detail, which will further facilitate the design of better geometric shapes. Although the proposed method has many advantages, there are still many problems that have not been solved, such as the reverse problem of parameters, how to extend the curve to the triangular domain, etc., which will be the future work.

\section{Acknowledgments}

This research was supported by National Natural Science Foundation of China (61861040), the Gansu education Department Science and Technology Achievement Transformation project (no. 2017D-09) and Gansu Province Science, technology project funding (no.17YF1FA119) and Lanzhou Science and Technology Plan Project (2018-4-35).

\section{Reference}

[1] G. Farin, Curves and Surfaces for Computer Aided Geometric Design, Academic Press, San Diego, 1993.

[2] J. Hoschek, D. Lasser, Fundamentals of Computer Aided Geometric Design, A.K. Peters, Wellesley, Ma, 1993.

[3] L. Piegl, W. Tiller, The NURBS book, Springer, New York, 1995.

[4] X.L. Han, Piecewise quartic polynomial curves with a shape parameters, J. Comput. Appl. Math. 195 (2006) 34-45.

[5] X.A. Han, Y.C. Ma, X.L. Huang, A novel generalization of Bezier curve and surface, J. Comput. Appl. Math. 217 (2008) $180-193$.

[6] L.L. Yan, J.F. Liang, A class of algebratic-trigonometric blended splines, J. Comput. Appl. Math. 235(2011) 2863-2879.

[7] L,L, Yan, J.F. Liang, An extension of the Bezier model, Appl. Math. Comput. 218(2011) 2863-2879.

[8] I. Juhasz, M. Hoffmann, On the quartic curve of Han, J. Comput. Math. 223(2009) 124-132.

[9] B.A. Barsky. Computer Graphics and Geometric Modelling Using Beta-splines, Springer, Heidelberg, 1988.

[10] J.A. Gregory, M. Sarfraz, A rational cubic spline with tension, Comput. Aided Geom. Design9 (1990) 1-13.

[11] P. Costantini, F. Pelosi, M. Sampoli, New spline spaces with generalized tension properties, BIT 48 (2008) 665-688

[12] P. Costantini, C. Manni, Geometric construction of spline curves with tension properties, Comput. Aided Geom. Design 20 (2003) 579-599.

[13] B.A. Barsky. Local control of bias and tension in Beta-splines, ACM Trans. Graphics 2 (1983) 109-134.

[14] R.J. Wu, G.H. Peng. Shape analysis of planar trigonometric Bezier curveswith two shape parameters, Int.J. Comput.Sci. 10(2013)441-447.

[15] P. Costantini, C. Manni, Geometric construction of spline curves with tension properties, Comput. Aided Geom. Design 20 (2003) 579-599.

[16] X.L. Han, A class of general quartic spline curves with shape parameters, Comput. Aided Geom. Design 28 (2011) $151-163$. 
[17] X.L. Han, Y.P. Zhu, Curve construction based on five trigonometric blending functions, BIT 52(2012) 953-979.

[18] P. Costantini, Curve and surface construction using variable degree polynomial splines, Comput. Aided Geom. Design 17 (2000) 419-466.

[19] M.L. Mazure, Quasi-Chebyshev splines with connexion matrices: application to variable degree polynomial splines, Comput. Aided Geom. Des. 18 (2001) 287-298.

[20] M.L. Mazure, Blossom and optimal bases, Adv. Comput. Math. 20(2004) 177-203.

[21] P. Costantini, T. Lyche, C. Manni, On a class of weak Tchebysheff systems, Numer. Math. 101(2005) 333-354.

[22] T. Bosnor, M. Rogina, Variable degree polynomial splines are Chebyshev splines, Adv. Comput. Math. 38 (2013) 383-400.

[23] Y.P. Zhu, X.L. Han, S.J. Liu, Curves construction based on four $\alpha \beta$-bernstein-like basis function, J. Comput. Appl. Math., 273(2015) $160-181$.

[24] K. Wang, G.C. Zhang, New trigonometric basis possessing denominator shape parameters, Mathe. Prol. In Engi, (2018) 1-25.

[25] M.L. Mazure, Which spaces for design, Numerische Mathematik, 110(2008) 357-392.

[26] M.L. Mazure, On dimension elevation in quasi extended Chebyshev spaces, Numerische Mathematik, 109(2008) $459-475$.

[27] M.L. Mazure, On a general new class of quasi Chebyshevian splines, Numerical Algorithms, 58(2011) 399-438.

[28] M.L. Mazure, Quasi Extended Chebyshev spaces and weight functions, Numerische Mathematik, 118(2011) 79-108.

[29] T. Bosner T, M. Rogina, Variable degree polynomial splines are Chebyshev splines, Advances in Computational Mathematics, $38(2013) 383-400$.

[30] J.M. Carnicer, J.M. Pena, Shape preserving representations and optimality of the Bernstein basis, Advances in Computational Mathematics, 1(1993) 173-196.

[31] J.M. Carnicer, J.M. Pena. Total positive bases for shape preserving curve design and optimality of B-splines, Computer Aided Geometric Design, 11(1994) 635-656.

[32] Y.P. Zhu, Research on theory and methods for geometric modeling based on basis functions possessing shape parameters, Changsha, Central South University, 2014.

[33] R.J. Wu, G.H. Peng, Shape analysis of planar trigonometric Bézier curves with two shape parameters, International Journal of Computer Science, 2(2013) 441-447.

[34] C.Y. Liu, Theory and application of convex curves and surfaces in CAGD, Enschede: Univercity of Twente, (2001)32-37.

[35] J.W. Bruce, P.J. Giblin, Curves and Singularities: A geometrical Introduction to singularity Theory, Cambridge University Press, 1993.

[36] W.Q. Shen, G.Z. Wang and P. Yin, Explicit representations of changeable degree spline basis functions, J. Comput. Appl. Math., 238 (2013) 39-50. 